\title{
On the relation between Volkov-Akulov and special conformal supersymmetry: a D3-brane perspective
}

\section{F. Coomans and B. Van Pol}

Instituut voor Theoretische Fysica, KU Leuven, Celestijnenlaan 200D B-3001 Leuven, Belgium

E-mail: frederik.coomans@fys.kuleuven.be, bert.vanpol@fys.kuleuven.be

ABSTRACT: We obtain the complete superconformal symmetry transformations on the worldvolume of a D3-brane in an $A d S_{5} \times S^{5}$ background by using a coset superspace approach. We show that in the large $R$-limit we recover all supersymmetries present on the worldvolume of a D3-brane in a Minkowski background, in particular the Volkov-Akulov supersymmetry. We conclude with a proposal for a scheme to construct higher derivative invariants in $D=4, \mathcal{N}=4$ settings.

KEYwords: Extended Supersymmetry, D-branes

ARXIV EPRINT: 1409.3749 


\section{Contents}

1 Introduction 1

2 Coset superspaces 3

2.1 Flat superspace 5

$2.2 A d S_{5} \times S^{5}$ superspace 6

3 D3-brane worldvolume theory 18

3.1 Local symmetries of the worldvolume actions 8

$\begin{array}{ll}3.2 & \text { The static gauge and the } Q \text {-gauge }\end{array}$

$\begin{array}{lll}3.3 & \text { D3-brane worldvolume in Minkowski background } & 10\end{array}$

3.4 D3-brane worldvolume in $A d S_{5} \times S^{5}$ background 11

4 From $A d S_{5} \times S^{5}$ to Minkowski: the large $R$ limit 12

5 Conclusions $\quad 14$

$\begin{array}{lr}\text { A Clifford algebras } & \mathbf{1 5}\end{array}$

$\begin{array}{lll}\text { A.1 The } \mathrm{SO}(2,4) \text { Clifford algebra } & 16\end{array}$

$\begin{array}{lll}\text { A.2 The } \mathrm{SO}(6) \text { Clifford algebra } & 16\end{array}$

$\begin{array}{lll}\text { A.3 The } \mathrm{SO}(1,9) \text { Clifford algebra } & 17\end{array}$

$\begin{array}{ll}\mathrm{B} \text { The } \mathrm{SU}(2,2 \mid 4) & \mathbf{1 7}\end{array}$

$\begin{array}{ll}\text { B.1 The AdS decomposition } & 19\end{array}$

$\begin{array}{ll}\text { B.2 The conformal decomposition } & 19\end{array}$

C $A d S_{5} \times S^{5}$ as a coset space $\quad 21$

C.1 $A d S_{5}$ as a coset space $\quad 21$

C.2 $S^{5}$ as a coset space 23

C.3 $A d S_{5} \times S^{5}$ and adapted fermionic coordinates 24

\section{Introduction}

In [1] new ways for constructing supersymmetric higher derivative invariants were investigated in settings where there are no known off-shell formulations. In particular, the action and supersymmetry transformation rules of the $D=4, \mathcal{N}=4$ Maxwell multiplet were deformed with higher derivative terms. This was done in such a way that at each order of the deformation the theory has 16 deformed Maxwell multiplet supersymmetries and 16 Volkov-Akulov (VA) type non-linear supersymmetries. The results were obtained by 
studying the worldvolume theory of the gauge-fixed D3-superbrane in a 10-dimensional Minkowski background.

It is still an open question if superconformal higher-derivative invariants in $D=4, \mathcal{N}=$ 4 supergravity exist. Constructing these invariants by using superconformal methods $[2-7]$ requires a superconformal extension of the rigid supersymmetric deformed Maxwell multiplet of [1]. A lot can be learned about such an extension by studying a D3-brane in an $A d S_{5} \times S^{5}$ background since the corresponding worldvolume theory is a superconformal one [8-10]. In this paper we aim to investigate the relation between the superconformal symmetry group of the $A d S_{5} \times S^{5}$ background and the VA supersymmetry group of the Minkowski background. We do this by applying the coset formalism [11-14], and constructing the transformation rules for the worldvolume theory of a D3-brane in these backgrounds. We investigate whether there is a way to mimic the superconformal symmetry induced by the $A d S_{5} \times S^{5}$ background in the case of the Minkowski background such that it could be used in the superconformal approach for constructing higher derivative terms. We choose a gauge for the worldvolume $\kappa$-symmetry that allows us to establish contact with the results of [1].

The paper is organized as follows. In section 2 we recap the coset formalism [11-14]. We apply it to both backgrounds, Minkowski and $A d S_{5} \times S^{5}$, to obtain the background super isometries. In section 3 we discuss the D3-brane worldvolume theory, in particular we look at the worldvolume symmetries, discuss how to gaugefix the local symmetries and discuss the effects of the different backgrounds. In section 4 we discuss the large $R$ limit that will allow us to compare the symmetry transformations of both backgrounds. In section 5 we present our conclusions and discuss a possible avenue for constructing higher derivative invariants. In appendix A we collect our conventions regarding Clifford matrices. In appendix B we present the $\mathrm{SU}(2,2 \mid 4)$ algebra as well as two ways of decomposing the $\mathrm{SO}(2,4)$ subalgebra, the AdS and conformal decompositions. Finally, in appendix $\mathrm{C}$ we provide details for the construction of the $A d S_{5} \times S^{5}$ background as a coset space.

Notational conventions. We use the following conventions for indices

\begin{tabular}{|c|c|}
\hline $\bar{M}$ & label for the coset generators in $\mathbf{K}$ \\
\hline $\bar{I}$ & label for the stability group generators in $\mathbf{H}$ \\
\hline$\Lambda=\{\bar{M}, \bar{I}\}$ & label for the generators of the superalgebra $\mathbf{G}=\mathbf{K} \oplus \mathbf{H}$ \\
\hline $\mathcal{A}$ & label for the collection of bosonic generators in $\mathbf{G}$ \\
\hline$\tilde{a}, \tilde{b}, \tilde{c}=0, \ldots, 4$ & $A d S_{5}$ tangent space indices \\
\hline$a, b, c=0, \ldots, 3$ & part of the $A d S_{5}$ tangent space indices such that $\tilde{a}=\{a, 4\}$ \\
\hline$a^{\prime}, b^{\prime}, c^{\prime}=5, \ldots, 9$ & $S^{5}$ tangent space indices \\
\hline$A, B, C=0, \ldots, 9$ & 10D tangent space indices such that $A=\left\{\tilde{a}, a^{\prime}\right\}$ \\
\hline$\tilde{m}, \tilde{n}, \tilde{p}=0, \ldots, 4$ & $5 \mathrm{D}$ spacetime indices, associated with the $A d S_{5}$ space \\
\hline$m, n, p=0, \ldots, 3$ & part of the $5 \mathrm{D}$ spacetime indices such that $\tilde{m}=\{m, 4\}$ \\
\hline$m^{\prime}, n^{\prime}, p^{\prime}=5, \ldots, 9$ & 5D spacetime indices, associated with the $S^{5}$ space \\
\hline
\end{tabular}




$$
\begin{array}{rl}
M, N, P=0, \ldots, 9 & \text { 10D spacetime indices such that }\left\{M=\tilde{m}, m^{\prime}\right\} \\
\alpha, \beta, \gamma=1, \ldots, 4 & \text { so }(2,4) \text { spinor indices projected } \\
& \text { on the righthanded chiral subspace }\left(A d S_{5}\right) \\
i, j, k=1, \ldots, 4 & \text { so(6) spinor indices projected } \\
& \text { on the righthanded chiral subspace }\left(S^{5}\right) \\
\hat{\alpha}, \hat{\beta}, \hat{\gamma}=1, \ldots, 32 & D=10 \text { Majorana-Weyl spinor indices } \\
I, J, K=1, \ldots 4 & \text { so(6) spinor indices } \\
\mu=0, \ldots 3 & \text { coordinate indices of the worldvolume of the D3-brane }
\end{array}
$$

\section{Coset superspaces}

In this section we briefly recap the formalism of Cartan forms on coset superspaces [11-14]. We then use this formalism to write down the superisometries of Minkowski superspace and $A d S_{5} \times S^{5}$ superspace.

We consider the coset manifold $G / H$, where $G$ is a supergroup and $H \subset G$ is a subgroup. Each coset is represented by a coset representative $\mathcal{G}(Z)$, labelled by supercoordinates $Z^{M}=\left\{X^{M}, \theta^{\alpha}\right\}$. Left-invariant Cartan 1-forms are defined as

$$
L(Z) \equiv \mathcal{G}(Z)^{-1} d \mathcal{G}(Z)
$$

Since $L(Z)$ is a group element close to the identity it is a $\mathbf{G}$ valued super 1-form

$$
L(Z)=L^{\Lambda} \mathbf{T}_{\Lambda}=d Z^{M} L_{M}^{\Lambda} \mathbf{T}_{\Lambda},
$$

where $\mathbf{T}_{\Lambda}$ are the generators of the superalgebra $\mathbf{G}$ associated to $G$.

We consider two decompositions which will be useful. First there is the coset decomposition of the algebra, defined by $\mathbf{G}=\mathbf{K} \oplus \mathbf{H}$ where $\mathbf{H}$ is the Lie-algebra associated with the stability group $H$ of $G, \mathbf{G}$ is the Lie-algebra of $G$, and $\mathbf{K}$ collects the coset generators. We introduce the split of labels $\Lambda=(\bar{M}, \bar{I})$, where $\bar{M}$ are the directions in $\mathbf{K}$ and $\bar{I}$ are the directions in $\mathbf{H}$. The second decomposition that we consider is a boson-fermion split of the algebra $\mathbf{G}=\mathbf{B} \oplus \mathbf{F}$, where $\mathbf{B}$ contains the bosonic generators $B_{A}$ and $\mathbf{F}$ the fermionic generators $F_{\alpha}$, and define the split of a $\mathbf{G}$-valued object $\mathrm{A}$ as

$$
A=A^{\Lambda} \mathbf{T}_{\Lambda}=A^{\mathbf{B}}+A^{\mathbf{F}}=A^{\mathcal{A}} \mathbf{T}_{\mathcal{A}}+A^{\alpha} \mathbf{F}_{\alpha}
$$

For the coset representative we choose the parametrization $\mathcal{G}(Z)=g(X) e^{\Theta}$, where $g(X)$ represents the bosonic coset representative of the coset space and

$$
\Theta=\Theta^{\alpha} \mathbf{F}_{\alpha}=\theta^{\dot{\alpha}} e_{\dot{\alpha}}^{\alpha}(X) \mathbf{F}_{\alpha},
$$

where $e_{\dot{\alpha}}{ }^{\alpha}(X)$ determines the choice of fermionic coordinates.

In $[11,12]$ the complete geometric superfields $L(Z)$ and Killing superfields $\Sigma(Z)$ for a generic maximally supersymmetric superspace were constructed independent of the choice 
of coordinates (to all orders in $\theta$ ), we repeat their results here. The Cartan 1-forms and the parameters $\Sigma$ (defining the superisometries) are split as follows

$$
\begin{aligned}
L & =E+\Omega=E^{\bar{M}} \mathbf{K}_{\bar{M}}+\Omega^{\bar{I}} \mathbf{H}_{\bar{I}}, \\
\Sigma & =\hat{\Xi}+\hat{\Lambda}=\hat{\Xi}^{\bar{M}} \mathbf{K}_{\bar{M}}+\hat{\Lambda}^{\bar{I}} \mathbf{H}_{\bar{I}},
\end{aligned}
$$

where the parameters $\left\{\hat{\Xi}^{\bar{M}}, \hat{\Lambda}^{\bar{I}}\right\}$ are defined in terms of the superisometries $\left\{\Xi^{M}, \Lambda^{\bar{I}}\right\}$ as

$$
\hat{\Xi}^{\bar{M}}=\Xi^{M} E_{M}^{\bar{M}}, \quad \hat{\Lambda}^{\bar{I}}=\Lambda^{\bar{I}}+\Xi^{M} \Omega_{M}^{\bar{I}} .
$$

We will be interested in maximally supersymmetric superspaces where $\mathbf{F} \subset \mathbf{K}$ or $\mathbf{F} \cap \mathbf{H}=$ 0. Both the Minkowski and $A d S_{5} \times S^{5}$ backgrounds fall in this category. The bosonic generators are split into $\mathbf{B}=\left\{\mathbf{P}_{a}, \mathbf{M}_{i}\right\}$, with $\mathbf{P}_{a} \in \mathbf{K}$ and $\mathbf{M}_{i} \in \mathbf{H}$. We also consider the gravitino $L_{0}^{\mathbf{F}}$ to be vanishing.

Splitting $\bar{M}$ into bosonic $a$ and fermionic $\alpha$, the supervielbein is given by [11, 12]

$$
E_{M}^{\bar{M}}=\left(\begin{array}{cc}
e_{\mu}{ }^{b}(X) & 0 \\
0 & e_{\dot{\alpha}}^{\beta}(X)
\end{array}\right)\left(\begin{array}{cc}
\delta_{b}{ }^{a}+(U A \mathcal{Y})_{b}{ }^{a} & (U A B)_{b}{ }^{\alpha} \\
(A \mathcal{Y})_{\beta}{ }^{a} & (A B)_{\beta}{ }^{\alpha}
\end{array}\right),
$$

where

$$
\begin{array}{ll}
A_{\alpha}{ }^{\beta}=2\left(\frac{\sinh ^{2} \mathcal{M} / 2}{\mathcal{M}^{2}}\right)_{\alpha}{ }_{\alpha}{ }^{\beta}, & B_{\alpha}{ }^{\beta}=(\mathcal{M} \operatorname{coth} \mathcal{M} / 2)_{\alpha}{ }^{\beta}, \\
\mathcal{Y}_{\alpha}{ }^{a}=-\Theta^{\delta} f_{\delta \alpha}{ }^{a}, & \mathcal{M}_{\alpha}{ }^{\beta}=f_{\alpha \gamma}{ }^{A} \Theta^{\gamma} \Theta^{\delta} f_{\delta A}{ }^{\beta},
\end{array}
$$

and $f_{\Lambda \Sigma} \Gamma^{\Gamma}$ are the structure constants of the algebra $\mathbf{G}$. The $e_{\mu}{ }^{a}$ form the vielbein of the bosonic space and $e_{\dot{\alpha}}^{\alpha}$ is the matrix introduced in the boson-fermion parametrization of the coset representative. The matrix $U_{a}{ }^{\alpha}$ and $\Theta^{\alpha}$ depend on the spinorial gauge choice $e_{\dot{\alpha}}{ }^{\beta}$

$$
U_{a}^{\alpha}=e_{a}^{\mu}\left[\theta^{\dot{\alpha}} \partial_{\mu} e_{\dot{\alpha}}^{\alpha}+\left(L_{0}^{A}\right)_{\mu} \theta^{\dot{\beta}} e_{\dot{\beta}}^{\beta} f_{A \beta}^{\alpha}\right] .
$$

The superisometries, $\Sigma(Z)=\mathcal{G}^{-1}(Z) \Upsilon_{0} \mathcal{G}(Z)$, in general are determined completely in terms of the $\theta=0$ Killing superfields $\Sigma_{0}^{\Lambda}$, which we denote here by

$$
\Sigma_{0}^{\Lambda} T_{\Lambda}=\tilde{\xi}^{a} \mathbf{P}_{a}+\tilde{\epsilon}^{\alpha} \mathbf{F}_{\alpha}+\tilde{l}^{i} \mathbf{M}_{i}
$$

where

$$
\tilde{\xi}^{a}=\xi^{\mu} e_{\mu}^{a}, \quad \tilde{\epsilon}^{\alpha}=\epsilon^{\dot{\alpha}} e_{\dot{\alpha}}^{\alpha}, \quad \tilde{l}^{i}=l^{i}+\xi^{\mu} \omega_{\mu}^{i} .
$$

In terms of the structure constants of $\mathbf{G}$, one can show $[11,12]$ that the superisometries are

$$
\begin{aligned}
& \Xi^{\mu}=\xi^{\mu}+\tilde{\epsilon}^{\beta}\left(\mathcal{M}^{-1} \tanh \mathcal{M} / 2\right)_{\beta}{ }^{\alpha} \mathcal{Y}_{\alpha}{ }^{a} e_{a}^{\mu}, \\
& \Xi^{\dot{\alpha}}=\left(\Theta^{\beta} \tilde{\xi}^{a} f_{a \beta}{ }^{\alpha}+\Theta^{\beta} \tilde{l}^{i} f_{i \beta}{ }^{\alpha}-\xi^{a} U_{a}{ }^{\alpha}\right) e_{\alpha}{ }^{\dot{\alpha}} \\
& \quad+\tilde{\epsilon}^{\beta}(\mathcal{M} \operatorname{coth} \mathcal{M})_{\beta}{ }^{\alpha} e_{\alpha}{ }^{\dot{\alpha}}-\tilde{\epsilon}^{\gamma}\left(\mathcal{M}^{-1} \tanh \mathcal{M} / 2\right)_{\gamma}{ }^{\beta}(\mathcal{Y} U)_{\beta}{ }^{\alpha} e_{\alpha}{ }_{\alpha}^{\alpha} .
\end{aligned}
$$

The variations of the superspace coordinates are given by

$$
\delta X^{\mu}=-\Xi^{\mu}, \quad \delta \theta^{\dot{\alpha}}=-\Xi^{\dot{\alpha}} .
$$

In the next subsections we will use equations (2.7) and (2.13) to write down the supervielbein and superisometries of the Minkowski and $A d S_{5} \times S^{5}$ background superspaces. 


\subsection{Flat superspace}

As a warm-up we derive the isometries and vielbein of the Minkowski background. We start from the super Poincaré group $G$. The algebra is given by

$$
\begin{aligned}
{\left[M_{A B}, M_{C D}\right] } & =\eta_{A[C} M_{D] B}-\eta_{B[C} M_{D] A}, \\
{\left[P_{A}, M_{B C}\right] } & =\eta_{A[B} P_{C]}, \\
{\left[M_{A B}, Q_{\hat{\alpha}}\right] } & =-\frac{1}{4}\left(\Gamma_{A B} Q\right)_{\hat{\alpha}}, \\
\left\{Q_{\hat{\alpha}}, Q_{\hat{\beta}}\right. & =\left(\Gamma^{A}\right)_{\hat{\alpha} \hat{\beta}} P_{A} .
\end{aligned}
$$

We make the split

$$
\mathbf{H}=\left\{M_{A B}\right\}, \quad \text { and } \quad \mathbf{K}=\left\{P_{A}, Q_{\hat{\alpha}}\right\} .
$$

This means that the indices of the previous section are chosen to be $\Lambda=\{A,[A B], \hat{\alpha}\}$, $\mathcal{A}=\{A,[A B]\}, \bar{I}=\{[A B]\}, \bar{M}=\{a, \hat{\alpha}\}$. The spacetime fields are given by

$$
e_{M}^{A}=\delta_{M}^{A}, \quad \psi_{M}=0, \quad \omega_{M}^{A B}=0,
$$

and the solutions to the spacetime Killing equations $(\theta=0)$ are

$$
\xi^{M}=a^{M}+\lambda_{(M)}^{M N} x_{N}, \quad \epsilon^{\hat{\alpha}}(x)=\varepsilon_{0}^{\hat{\alpha}}, \quad l^{A B}=\lambda_{(M)}^{M N} \delta_{M}^{A} \delta_{N}^{B},
$$

where $a^{M}, \lambda_{(M)}^{M N}$ and $\varepsilon_{0}^{\hat{\alpha}}$ are constant parameters. The matrix $\mathcal{M}$ vanishes, and the matrix $e_{\dot{\hat{\alpha}}}^{\hat{\beta}}=\delta_{\dot{\hat{\alpha}}}^{\hat{\beta}}$. The supervielbein (2.7) is then given by

$$
E^{\hat{\alpha}}=d \theta^{\hat{\alpha}}, \quad E^{A}=d x^{A}+\bar{\theta} \hat{\Gamma}^{A} d \theta,
$$

where we suppressed the spinor indices in $\bar{\theta} \hat{\Gamma}^{A} d \theta=\theta^{\alpha}\left(\hat{\Gamma}^{A}\right)_{\alpha}^{\beta} d \theta_{\beta}$.

Plugging everything in (2.12), we obtain the well-known superisometries

$$
\begin{aligned}
\delta x^{M} & =-\Xi^{M}=-a^{M}-\lambda_{(M)}^{M N} x_{N}-\frac{1}{2}\left(\bar{\varepsilon}_{0} \hat{\Gamma}^{M} \theta+\text { h.c. }\right), \\
\delta \theta^{\hat{\alpha}} & =-\Xi^{\hat{\alpha}}=-\varepsilon_{0}^{\hat{\alpha}}-\frac{1}{4} \lambda_{(M)}^{M N}\left(\hat{\Gamma}_{M N} \theta\right)^{\hat{\alpha}} .
\end{aligned}
$$

To facilitate things later, we introduce projectors $\mathcal{P}_{Q, S}=\frac{1}{2}\left(1 \mp \gamma_{5}\right) \otimes I_{8}$ (this is similar to what we will do for the $A d S_{5} \times S^{5}$ case (see also appendix B)) such that

$$
\mathcal{P}_{Q} \theta=\theta_{\alpha}^{i}, \quad \mathcal{P}_{S} \theta=\vartheta_{\alpha}^{i},
$$

and we make a similar split for $\varepsilon_{0}$ into $\epsilon_{\alpha}^{i}$ and $\eta_{\alpha}^{i}$ respectively. In terms of these refined variables, we have for the transformations

$$
\begin{gathered}
\delta x^{M}=-a^{M}-\lambda_{(M)}^{M N} x_{N}-\frac{1}{2}\left[\left(\bar{\epsilon}_{i} \gamma^{m} \theta^{i}+\bar{\eta}_{i} \gamma^{m} \vartheta^{i}\right) \delta_{m}^{M}+\left(\bar{\epsilon}_{i} \vartheta^{i}-\bar{\eta}_{i} \theta^{i}\right) \delta_{4}^{M}+\left(\bar{\epsilon}_{i} \vartheta^{j}\right.\right. \\
\left.\left.+\bar{\eta}_{i} \theta^{j}\right)\left(\gamma^{\prime m^{\prime}}\right)_{j}^{i} \delta_{m^{\prime}}^{M}+\text { h.c. }\right]
\end{gathered}
$$




$$
\begin{aligned}
\delta \theta^{i}=-\epsilon^{i}- & \frac{1}{4} \lambda_{(M)}^{m n} \gamma_{m n} \theta^{i}-\frac{1}{4} \lambda_{(M)}^{m^{\prime} n^{\prime}}\left(\gamma_{m^{\prime} n^{\prime}}^{\prime}\right)_{j}{ }^{i} \theta^{j}+\frac{1}{2} \lambda_{(M)}^{m 4} \gamma_{m} \vartheta^{i}-\frac{1}{2} \lambda_{(M)}^{m^{\prime} 4}\left(\gamma_{m^{\prime}}^{\prime}\right)_{j}{ }^{i} \theta^{j} \\
& -\frac{1}{4} \lambda_{(M)}^{m n^{\prime}} \gamma_{m}\left(\gamma_{n^{\prime}}^{\prime}\right)_{j}{ }_{j} \vartheta^{j}, \\
\delta \vartheta^{i}=-\eta^{i} & -\frac{1}{4} \lambda_{(M)}^{m n} \gamma_{m n} \vartheta^{i}-\frac{1}{4} \lambda_{(M)}^{m^{\prime} n^{\prime}}\left(\gamma_{m^{\prime} n^{\prime}}^{\prime}\right)_{j}{ }^{i} \vartheta^{j}-\frac{1}{2} \lambda_{(M)}^{m 4} \gamma_{m} \theta^{i}+\frac{1}{2} \lambda_{(M)}^{m^{\prime} 4}\left(\gamma_{m^{\prime}}^{\prime}\right)_{j}{ }_{j} \vartheta^{j} \\
& -\frac{1}{4} \lambda_{(M)}^{m n^{\prime}} \gamma_{m}\left(\gamma_{n^{\prime}}^{\prime}\right)_{j}{ }^{i} \theta^{j} .
\end{aligned}
$$

This form of the isometries will be used to compare with the large $R$ limit of the $A d S_{5} \times S^{5}$ isometries. For the $A d S_{5} \times S^{5}$ background, however, there is no mixing between the first five and last five directions, this means that $\lambda_{(M)}^{m n^{\prime}}=\lambda_{(M)}^{4 n^{\prime}}=0$. For this reason we will set these equal to zero from here on out.

\section{$2.2 \quad A d S_{5} \times S^{5}$ superspace}

To construct this superspace we start from the superconformal group $G=\mathrm{SU}(2,2 \mid 4)$, which has $\mathrm{SO}(4,2) \times \mathrm{SO}(6)$ as its bosonic subgroup. The superalgebra is presented in more detail in appendix B. For this supercoset the stability group $H$ is the product group $\mathrm{SO}(4,1) \times$ $\mathrm{SO}(5)$, which is purely bosonic. The $30+32$ generators of $\mathrm{SU}(2,2 \mid 4) \supset \mathrm{SO}(4,2) \times \mathrm{SO}(6)$ are decomposed into $5+5$ translations $\tilde{P}_{\tilde{m}}$ and $P_{m^{\prime}}^{\prime}, 10+10$ Lorentz generators $\tilde{M}_{\tilde{m} \tilde{n}}$ and $M_{m^{\prime} n^{\prime}}^{\prime}$, and $16+16$ supersymmetries $Q_{\alpha}{ }^{i}$ and $S_{\alpha}{ }^{i}$. This superspace has (10|32) coordinates ( 5 coordinates $x^{\tilde{m}}=\left\{x^{m}, \rho\right\}$ of AdS, 5 coordinates $z^{m^{\prime}}$ of the sphere and 32 fermionic coordinates $\theta_{\alpha}$ and $\vartheta_{\alpha}{ }^{i}$ ). We have made the split

$$
\mathbf{H}=\left\{\tilde{M}_{\tilde{m} \tilde{n}}, M_{m^{\prime} n^{\prime}}^{\prime}\right\}, \quad \mathbf{K}=\left\{\tilde{P}_{\tilde{m}}, P_{m^{\prime}}^{\prime}, Q_{\alpha}^{i}, S_{\alpha}{ }^{i}\right\} .
$$

This supercoset is an example of a maximal supersymmetric coset, i.e. all fermionic generators are in $\mathbf{K}$. We refer to appendix $\mathrm{C}$ for a detailed discussion of the construction of the bosonic part of this coset space. Appendix $\mathrm{C}$ also contains a discussion on the choice of fermionic coordinates $e_{\hat{\alpha}}^{\hat{\beta}}$ and the choice we make is given in equation (C.26). In this section we will combine the results of appendix $\mathrm{C}$ and construct the supervielbein and superspace isometries (pushing the details of the coset construction to appendix C).

The metric of $A d S_{5} \times S^{5}$ is given by the sum of (C.2) and (C.18)

$$
d s^{2}=\rho^{2} d x^{2}+\left(\frac{R}{\rho}\right)^{2} d \rho^{2}+\frac{4 R^{2}}{\left(1+z^{2}\right)^{2}} d z^{2} .
$$

The supervielbein (2.5) of the geometry

$$
E=E^{\bar{M}} K_{\bar{M}}=E^{m} \tilde{P}_{m}+E^{\rho} \tilde{P}_{\rho}+E^{m^{\prime}} P_{m^{\prime}}^{\prime}+\left(\bar{Q}_{i} E_{Q}^{i}+\text { h.c. }\right)+\left(\bar{S}_{i} E_{S}^{i}+\text { h.c. }\right),
$$

has components

$$
\begin{gathered}
E^{m}=\rho\left[d x^{n}\left(\delta_{n}{ }^{m}-\frac{1}{2}\left(\frac{R}{\rho}\right)^{2} \bar{\vartheta}_{i} \gamma_{n} \vartheta^{j} \bar{\vartheta}_{j} \gamma^{m} \vartheta^{i}\right)+\left(\frac{1}{2} d \bar{\theta}_{i} \gamma^{m} \theta^{i}+\frac{1}{4} \bar{\theta}_{i} d \vartheta^{j} \bar{\theta}_{j} \gamma^{m} \theta^{i}+\text { h.c. }\right)\right. \\
\left.+\left(\frac{R}{\rho}\right)^{2}\left(\frac{1}{2} d \bar{\vartheta}_{i} \gamma^{m} \vartheta^{i}+\frac{1}{4} \bar{\vartheta}_{i} d \theta^{j} \bar{\vartheta}_{j} \gamma^{m} \vartheta^{i}+\text { h.c. }\right)\right]+\mathcal{O}(\theta \wedge \vartheta),
\end{gathered}
$$




$$
\begin{aligned}
E^{\rho} & =\frac{R}{\rho}\left[d \rho-\frac{1}{2}\left(d \bar{\theta}_{i} \vartheta^{i}-d \bar{\vartheta}_{i} \theta^{i}+\text { h.c. }\right) \rho\right]+\mathcal{O}(\theta \wedge \vartheta), \\
E^{m^{\prime}} & =e^{m^{\prime}}-\frac{R}{2}\left(d \bar{\theta}_{i} \vartheta^{j}+d \bar{\vartheta}_{i} \theta^{j}+d x^{m} \bar{\vartheta}_{i} \gamma_{m} \vartheta^{j}+\text { h.c. }\right)\left(u \gamma^{\prime m^{\prime}} u^{-1}\right)_{j}^{i}+\mathcal{O}(\theta \wedge \vartheta), \\
E_{Q}^{i} & =\rho^{1 / 2}\left[d \theta^{j}-d x^{m} \gamma_{m} \vartheta^{j}+\frac{1}{3} \theta^{k}\left(d \bar{\vartheta}_{k} \theta^{j}-\bar{\theta}_{k} d \vartheta^{j}\right)\right] u_{j}{ }^{i}+\mathcal{O}(\theta \wedge \vartheta), \\
E_{S}^{i} & =\rho^{-1 / 2}\left[d \vartheta^{j}+\frac{1}{3}\left(2 d \bar{\theta}_{k} \vartheta^{j}-\bar{\vartheta}_{k} d \theta^{j}\right)+d x^{m} \vartheta^{k} \bar{\vartheta}_{k} \gamma_{m} \vartheta^{j}\right] u_{j}{ }^{i}+\mathcal{O}(\theta \wedge \vartheta) .
\end{aligned}
$$

Here $\mathcal{O}(\theta \wedge \vartheta)$ stands for terms containing both $\theta^{i}$ and $\vartheta^{i}$. We do not include these terms because they will drop out when we discuss the D3-brane embedding and gauge-fixing in section 3 , where our gauge choice will set $\theta^{i}=0$. We have left the coordinates of the sphere unspecified here. They are coded in the coset representative $u$ and given in appendix C.2.

The superisometries for the various coordinates are

$$
\begin{aligned}
\delta x^{m}= & -\xi_{C}^{m}(x)-\frac{1}{2}\left(\bar{\epsilon}_{i}(x) \gamma^{m} \theta^{i}+\text { h.c. }\right)-\frac{1}{4}\left(\bar{\eta}_{i} \theta^{j} \bar{\theta}_{j} \gamma^{m} \theta^{i}+\text { h.c. }\right) \\
& -\left(\frac{R}{\rho}\right)^{2}\left[\lambda_{(K)}^{m}+\frac{1}{2}\left(\bar{\eta}_{i} \gamma^{m} \vartheta^{i}+\text { h.c. }\right)+\frac{1}{4}\left(\bar{\epsilon}_{i}(x) \vartheta^{j} \bar{\vartheta}_{j} \gamma^{m} \vartheta^{i}+\text { h.c. }\right)\right] \\
& +\mathcal{O}(\theta \wedge \vartheta), \\
\delta \rho= & \Lambda_{D}(x) \rho-\frac{1}{2}\left(\bar{\epsilon}_{i}(x) \vartheta^{i}-\bar{\eta}_{i} \theta^{i}+\text { h.c. }\right) \rho+\mathcal{O}(\theta \wedge \vartheta) \\
\delta z^{m^{\prime}}= & -\xi^{m^{\prime}}(z)+\frac{\left(1-z^{2}\right)}{4}\left(\bar{\epsilon}_{i}(x) \vartheta^{j}+\bar{\eta}_{i} \theta^{j}+\text { h.c. }\right)\left(u \gamma^{\prime m^{\prime}} u^{-1}\right)_{j}^{i}+\mathcal{O}(\theta \wedge \vartheta), \\
\delta \theta^{i}= & -\epsilon^{i}(x)-\frac{1}{2} \Lambda_{D}(x) \theta^{i}-\frac{1}{4} \Lambda_{M}(x) \cdot \gamma \theta^{i}-\frac{1}{4} \theta^{j} \Lambda_{\mathrm{SO}(6)}^{I J}\left(\hat{\gamma}_{I J}^{\prime}\right)_{j}{ }^{i} \\
& -\left(\frac{R}{\rho}\right)^{2}\left[\lambda_{(K)}^{m}+\frac{1}{2}\left(\bar{\eta}_{j} \gamma^{m} \vartheta^{j}+\text { h.c. }\right)+\frac{1}{4}\left(\bar{\epsilon}_{j}(x) \vartheta^{k} \bar{\vartheta}_{k} \gamma^{m} \vartheta^{j}+\text { h.c. }\right)\right] \gamma_{m} \vartheta^{i} \\
& -\frac{2}{3} \theta^{j}\left(2 \bar{\eta}_{j} \theta^{i}-\bar{\theta}_{j} \eta^{i}\right)+\mathcal{O}(\theta \wedge \vartheta), \\
\delta \vartheta^{i}= & -\eta^{i}+\lambda_{(K)}^{m} \gamma_{m} \theta^{i}+\frac{1}{2} \Lambda_{D}(x) \vartheta^{i}-\frac{1}{4} \Lambda_{M}(x) \cdot \gamma \vartheta^{i}-\frac{1}{4} \vartheta^{j} \Lambda_{\mathrm{SO}(6)}^{I J}\left(\hat{\gamma}_{I J}^{\prime}\right)_{j}{ }_{j} \\
& -\frac{2}{3} \vartheta^{j}\left(2 \bar{\epsilon}_{j}(x) \vartheta^{i}-\bar{\vartheta}_{j} \epsilon^{i}(x)\right)+\mathcal{O}(\theta \wedge \vartheta)
\end{aligned}
$$

These $A d S_{5} \times S^{5}$ isometries have been written in terms of $x$-dependent combinations of the superconformal parameters $a^{m}, \lambda_{(M)}^{m n}, \lambda_{(K)}^{m}$ and $\lambda_{D}$ as defined in (C.14). We have defined

$$
\epsilon^{i}(x)=\epsilon+x^{m} \gamma_{m} \eta^{i}
$$

and the supersymmetries and special supersymmetries are parametrized by $\epsilon$ and $\eta$. $\Lambda_{\mathrm{SO}(6)}^{I J}$ are the parameters of the $\mathrm{SO}(6)$ R-symmetry, $\xi^{m^{\prime}}(z)$ is given in (C.24), and $\hat{\gamma}_{I J}^{\prime}$ are elements of the 6-dimensional Clifford algebra, realizing the translation between $\mathrm{SO}(6)$ and $\mathrm{SU}(4)$,

$$
\Lambda_{\mathrm{SO}(6)}^{I J}=\frac{1}{2} \Lambda_{\mathrm{SU}(4) i}{ }^{j}\left(\hat{\gamma}^{\prime I J}\right)_{j}{ }^{i} .
$$




\section{D3-brane worldvolume theory}

The world-volume action of a generic super D3-brane probe consists of two parts [15-17]

$$
S=S_{\mathrm{DBI}}+S_{\mathrm{WZ}} .
$$

The worldvolume $\mathcal{M}_{4}$ is parametrized by 4 coordinates $\sigma^{\mu}$.

The first term of the action, $S_{\mathrm{DBI}}$, can be written as

$$
S_{\mathrm{DBI}}=-\frac{1}{\alpha^{2}} \int_{\mathcal{M}_{4}} d^{4} \sigma \sqrt{-\operatorname{det}\left(G_{\mu \nu}+\alpha \mathcal{F}_{\mu \nu}\right)}
$$

It contains the induced metric

$$
G_{\mu \nu}=E_{\mu}^{a} \eta_{a b} E_{\nu}^{b}, \quad E_{\mu}^{a}=\partial_{\mu} Z^{M} E_{M}^{a},
$$

where $E_{\mu}^{a}$ is the pull-back of the background vielbein $E^{a}$ to the worldvolume. $\alpha^{2}$ corresponds to the inverse brane tension and $\mathcal{F}_{\mu \nu}$ depends on the other fields on the worldvolume. The superspace coordinates are now fields on the worldvolume $Z^{M}=Z^{M}(\sigma)$.

The Wess-Zumino component is an integral over the worldvolume of an appropriate 4form $\mathcal{A}_{4}$. It can be written as a closed 5-form over a 5-dimensional manifold which has the worldvolume as its boundary, and whose leading term is the pull-back of the background 5 superform. It contains further terms that describe interaction of the extra tensor fields with the background forms of lower order.

Both terms of the brane action are by construction (separately) invariant under the background superisometries. The background isometries are now symmetries acting on fields, i.e. they depend on the worldvolume coordinates $\sigma$ through $Z^{M}(\sigma)$. Upon fixing the embedding of the brane in the background, the rigid background isometries will be realized on the remaining world-volume fields.

\subsection{Local symmetries of the worldvolume actions}

The D3-brane actions not only have global symmetries due to the background isometries, they also come with local symmetries. The first set of local symmetries of this action are the world-volume diffeomorphisms. They act as Lie-derivatives on the fields

$$
\delta_{\text {loc.diff. }} Z^{M}=\zeta^{\mu}(\sigma) \partial_{\mu} Z^{M}, \quad \delta_{\text {loc.diff. }} \mathcal{F}_{\mu \nu}=\zeta^{\rho}(\sigma) \partial_{\rho} \mathcal{F}_{\mu \nu}-2 \partial_{[\mu} \zeta^{\rho}(\sigma) \mathcal{F}_{\nu] \rho} .
$$

The second local symmetry is called $\kappa$-symmetry $[16,17]$, which is a local fermionic symmetry. Its parameter is a 10-dimensional spinor $\kappa$, depending on the worldvolume coordinates. The variations $\delta Z^{M}$ of the world-volume fields are defined in terms of the supervielbein by

$$
\begin{aligned}
\delta_{\kappa} E^{a} & \equiv\left(\delta_{\kappa} Z^{M}\right) E_{M}^{a}=0 \\
\delta_{\kappa} E^{\alpha} & \equiv\left(\delta_{\kappa} Z^{M}\right) E_{M}^{\alpha}=\kappa^{\beta}(\sigma)\left(1+\Gamma^{\mathcal{C}}\right)_{\beta}{ }^{\alpha} .
\end{aligned}
$$

The matrix $\Gamma$ appears here as its charge conjugate $\Gamma^{\mathcal{C}}$ and it is an element of the 10dimensional Clifford algebra, satisfying $\Gamma^{2}=1, \operatorname{Tr} \Gamma=0$. It is a combination of gamma matrices and depends on the worldvolume fields. For the probe $D 3$-brane, $\Gamma$ is given by

$$
\Gamma=\left(\begin{array}{cc}
0 & \beta_{-} \\
-\beta_{+} & 0
\end{array}\right),
$$


with

$$
\begin{aligned}
& \beta_{-}=\frac{1}{\sqrt{-\operatorname{det}\left(G_{\mu \nu}+\alpha \mathcal{F}_{\mu \nu}\right)}}\left(\sum_{k=0}^{2} \frac{(-\alpha)^{k}}{2^{k} k !} \gamma^{\mu_{1} \nu_{1} \ldots \mu_{k} \nu_{k}} \mathcal{F}_{\mu_{1} \nu_{1}} \ldots \mathcal{F}_{\mu_{k} \nu_{k}}\right) \Gamma^{D_{3},} \\
& \beta_{+}=\frac{1}{\sqrt{-\operatorname{det}\left(G_{\mu \nu}+\alpha \mathcal{F}_{\mu \nu}\right)}}\left(\sum_{k=0}^{2} \frac{\alpha^{k}}{2^{k} k !} \gamma^{\mu_{1} \nu_{1} \ldots \mu_{k} \nu_{k}} \mathcal{F}_{\mu_{1} \nu_{1}} \ldots \mathcal{F}_{\mu_{k} \nu_{k}}\right) \Gamma^{D_{3}},
\end{aligned}
$$

where $\Gamma^{D_{3}}=\frac{1}{4 !} \epsilon^{\mu \nu \rho \sigma} \gamma_{\mu \nu \rho \sigma}$ and $\gamma^{\mu}$ are the pullback of the 10-dimensional gamma matrices.

We can invert the relations (3.5) to $\delta_{\kappa} X^{\mu}$ and $\delta_{\kappa} \theta^{\alpha}$ by using the inverse vielbein $[11,12]$

$$
\begin{aligned}
\delta_{\kappa} X^{\mu} & =-\kappa^{\beta}\left(1+\Gamma^{\mathcal{C}}\right)_{\beta}^{\alpha}\left(\mathcal{M}^{-1} \tanh \frac{\mathcal{M}}{2} \Upsilon\right)_{\alpha}{ }^{a} e_{a}^{\mu}, \\
\delta_{\kappa} \theta^{\dot{\alpha}} & =\kappa^{\gamma}\left(1+\Gamma^{\mathcal{C}}\right)_{\gamma}^{\beta}\left(\mathcal{M} \sinh ^{-1} \mathcal{M}+\mathcal{M}^{-1} \tanh \frac{\mathcal{M}}{2} \Upsilon U\right)_{\beta}{ }^{\alpha} e_{\alpha}^{\dot{\alpha}} .
\end{aligned}
$$

Comparing with (2.12) we see that they almost act as supersymmetries, the difference being in the higher order fermion terms in $\delta_{\kappa} \theta^{\dot{\alpha}}$.

The irreducible $\kappa$ symmetries are defined by the algebraic constraint

$$
\left(1-\Gamma_{\mathrm{cl}}\right) \kappa=0,
$$

where $\Gamma_{\mathrm{cl}}$ is the value of $\Gamma$ at the classical value of the fields, compatible with the gauge fixing and brane wave equations. We can write the irreducible $\kappa$ symmetries as

$$
\kappa_{+} \equiv(1+\Gamma) \kappa_{*},
$$

where $\kappa_{*}$ is a solution to equation $(3.9),(1-\Gamma) \kappa_{*}=0$.

\subsection{The static gauge and the $Q$-gauge}

The embedding of the brane in the background can be described by identifying some of the worldvolume coordinates with the spacetime coordinates of the background. This 'gauge fixing' has to be admissible, which means that it has to be compatible with the equations of motion derived from the probe-brane action, the branewave equations. We will consider an infinite extended brane and will therefore take the static gauge

$$
\sigma^{\mu}=\delta_{m}^{\mu} x^{m}
$$

where $x^{m}$ are 4 coordinates of the background geometry. This gauge will only yield a stable configuration in specific backgrounds [9]. Two examples are the flat background and the $A d S \times S$ background where the $x^{m}$ have to be the directions parallel to the boundary of AdS. The full transformation of the fields $Z^{M}(\sigma)$ is

$$
\delta Z^{M}=\zeta^{\mu} \partial_{\mu} Z^{M}+\delta_{\text {global }} Z^{M}+\delta_{\kappa} Z^{M},
$$

where $\delta_{\text {global }} Z^{M}$ are the transformations in (2.19) or (2.26). In order to preserve the gauge choice (3.11) we need to impose the condition $\delta x^{m}=0$, leading to a decomposition law for $\zeta^{\mu}$. 
In fixing the $\kappa$-symmetry we will be guided by the effects of the $A d S_{5} \times S^{5}$ background. There are two natural ways to gauge-fix the $\kappa$-symmetry and get rid of half of the fermionic gauge-degrees of freedom on the worldvolume. We can either set $\vartheta^{i}=0$ (S-gauge) or we can set $\theta^{i}=0$ ( $Q$-gauge). However, the $S$-gauge is not admissible for the infinite static branes in their own near-horizon geometry. The classical values of the fields in the static gauge are $x^{m}=\delta_{\mu}^{m} \sigma^{\mu}, \rho=$ constant, $z^{m^{\prime}}=$ constant, $\theta^{i}=\vartheta^{i}=0, \mathcal{F}_{\mu \nu}=0$ leading to $\Gamma_{c l}=\hat{\gamma}_{S T}$, where this matrix $\hat{\gamma}_{S T}$ is precisely the one used in the projector to define $Q$ and $S$ supersymmetry (Appendix B.2). This means that a gauge-fixing

$$
0=\vartheta^{i}=\frac{1}{2}\left(1-\hat{\gamma}_{S T}\right) \Theta^{i}
$$

will not affect the irreducible $\kappa$ symmetry and is not admissible. Since we are interested in the $A d S_{5} \times S^{5}$ background, this leaves us with the 'natural' choice of the $Q$-gauge, $\theta^{i}=0$. Imposing this condition will leave us with a decomposition law for $\kappa_{+}$.

\subsection{D3-brane worldvolume in Minkowski background}

We consider the embedding of a D3-brane in a Minkowski background. The $\kappa$-symmetry transformation rules (3.8) become

$$
\begin{aligned}
& \delta_{\kappa} x^{M}=-\frac{1}{2}\left[\left(\bar{\kappa}_{+Q i} \gamma^{m} \theta^{i}+\bar{\kappa}_{+S i} \gamma^{m} \vartheta^{i}\right) \delta_{m}^{M}+\left(\bar{\kappa}_{+Q i} \vartheta^{i}-\bar{\kappa}_{+S i} \theta^{i}\right) \delta_{4}^{M}\right. \\
&\left.+\left(\bar{\kappa}_{+Q i} \vartheta^{j}+\bar{\kappa}_{+S i} \theta^{j}\right)\left(\gamma^{\prime m^{\prime}}\right)_{j}^{i} \delta_{m^{\prime}}^{M}+\text { h.c. }\right], \\
& \delta_{\kappa} \theta^{i}=\kappa_{+Q}^{i}, \quad \delta_{\kappa} \vartheta=\kappa_{+S}^{i},
\end{aligned}
$$

where we have introduced the projections $\mathcal{P}_{Q, S} \kappa_{+}=\kappa_{+Q, S}$.

As discussed in the previous section, the condition $\delta x^{m}=0$, needed to preserve the static gauge, and the $Q$-gauge condition $\theta^{i}=0$ (fixing the kappa gauge) give us two decomposition laws (up to cubic fermion terms)

$$
\kappa_{+Q}^{i}=\epsilon^{i}-\frac{1}{2} \lambda^{m 4} \gamma_{m} \vartheta^{i}
$$

and

$$
\zeta^{\mu}(\sigma)=a^{\mu}+\lambda_{(M)}^{\mu N} x_{N}-\frac{1}{2}\left[\bar{\vartheta}_{i} \gamma^{\mu}\left(\eta^{i}-\beta_{+}\left(\epsilon^{i}-\frac{1}{2} \lambda^{n 4} \gamma_{n} \vartheta^{i}\right)\right)+\text { h.c. }\right] .
$$

The remaining fields then have as transformation laws

$$
\begin{aligned}
\delta x^{4}= & \xi^{\mu} \partial_{\mu} x^{4}-\frac{1}{2}\left[\bar{\vartheta}_{i} \gamma^{\mu}\left(\eta^{i}-\beta_{+}\left(\epsilon^{i}-\frac{1}{2} \lambda^{n 4} \gamma_{n} \vartheta^{i}\right)\right)+\text { h.c. }\right] \partial_{\mu} x^{4} \\
- & \xi^{4}-\left[\bar{\vartheta}_{i}\left(\epsilon^{i}-\frac{1}{4} \lambda^{m 4} \gamma_{m} \vartheta^{i}\right)+\text { h.c. }\right], \\
\delta x^{m^{\prime}}= & \xi^{\mu} \partial_{\mu} x^{m^{\prime}}-\frac{1}{2}\left[\bar{\vartheta}_{i} \gamma^{\mu}\left(\eta^{i}-\beta_{+}\left(\epsilon^{i}-\frac{1}{2} \lambda^{n 4} \gamma_{n} \vartheta^{i}\right)\right)+\text { h.c. }\right] \partial_{\mu} x^{m^{\prime}} \\
& -\xi^{m^{\prime}}-\left[\left(\bar{\vartheta}_{i} \epsilon^{j}-\frac{1}{4} \lambda^{m 4} \vartheta_{i} \gamma_{m} \vartheta^{j}\right)\left(\gamma^{m^{\prime}}\right)_{j}^{i}+\text { h.c. }\right],
\end{aligned}
$$




$$
\begin{aligned}
\delta \vartheta^{i}= & \xi^{\mu} \partial_{\mu} \vartheta^{i}-\frac{1}{2}\left[\bar{\vartheta}_{j} \gamma^{\mu}\left(\eta^{j}-\beta_{+}\left(\epsilon^{j}-\frac{1}{2} \lambda^{n 4} \gamma_{n} \vartheta^{j}\right)\right)+\text { h.c. }\right] \partial_{\mu} \vartheta^{i} \\
& -\frac{1}{4} \lambda_{(M)}^{m n} \gamma_{m n} \vartheta^{i}-\frac{1}{4} \lambda_{(M)}^{m^{\prime} n^{\prime}}\left(\gamma_{m^{\prime} n^{\prime}}^{\prime}\right){ }_{j}{ }^{i} \vartheta^{j}-\left[\eta^{i}+\beta_{+}\left(\epsilon^{i}-\frac{1}{2} \lambda^{m 4} \gamma_{m} \vartheta^{i}\right)\right]
\end{aligned}
$$

where we used that $\kappa_{+S}=-\beta_{+} \kappa_{+Q}$ and defined $\xi^{M}=a^{M}+\lambda_{(M)}^{M N} x_{N}$.

\subsection{D3-brane worldvolume in $A d S_{5} \times S^{5}$ background}

In this section we consider the D3-brane embedded in its own near-horizon background, $A d S_{5} \times S^{5}$. Embedding a D3-brane in this background, the background coordinates are promoted to worldvolume fields and their transformations under $\kappa$ symmetry (3.8) are given by

$$
\begin{aligned}
\delta_{\kappa} x^{m}= & -\frac{1}{2}\left(\bar{\kappa}_{+Q i} \gamma^{m} \theta^{i}+\text { h.c. }\right)-\frac{1}{4}\left(\bar{\kappa}_{+S i} \theta^{j} \bar{\theta}_{j} \gamma^{m} \theta^{i}+\text { h.c. }\right) \\
& -\left(\frac{R}{\rho}\right)^{2}\left[\frac{1}{2}\left(\bar{\kappa}_{+S i} \gamma^{m} \vartheta^{i}+\text { h.c. }\right)+\frac{1}{4}\left(\bar{\kappa}_{+Q i} \vartheta^{j} \bar{\vartheta}_{j} \gamma^{m} \vartheta^{i}+\text { h.c. }\right)\right]+\mathcal{O}(\theta \wedge \vartheta) \\
\delta_{\kappa} \theta^{i}= & \kappa_{+Q}^{i}-\left(\frac{R}{\rho}\right)^{2}\left[\frac{1}{2}\left(\bar{\kappa}_{+S j} \gamma^{m} \vartheta^{j}+\text { h.c. }\right)+\frac{1}{4}\left(\bar{\kappa}_{+Q j} \vartheta^{k} \bar{\vartheta}_{k} \gamma^{m} \vartheta^{j}+\text { h.c. }\right)\right] \gamma_{m} \vartheta^{i} \\
& -\frac{1}{3} \theta^{j}\left(2 \bar{\kappa}_{+S j} \theta^{i}-\bar{\theta}_{j} \kappa_{+S}^{i}\right)+\mathcal{O}(\theta \wedge \vartheta), \\
\delta_{\kappa} \vartheta^{i}= & \kappa_{+S}^{i}-\frac{1}{3} \vartheta^{j}\left(2 \bar{\kappa}_{+Q j} \vartheta^{i}-\bar{\vartheta}_{j} \kappa_{+Q}^{i}\right)+\mathcal{O}(\theta \wedge \vartheta), \\
\delta_{\kappa} \rho= & -\frac{1}{2}\left(\bar{\kappa}_{+Q i} \vartheta^{i}-\bar{\kappa}_{+S i} \theta^{i}\right) \rho+\text { h.c }+\mathcal{O}(\theta \wedge \vartheta), \\
\delta_{\kappa} z^{m^{\prime}}= & \frac{\left(1-z^{2}\right)}{4}\left(\bar{\kappa}_{+Q i} \vartheta^{j}+\bar{\kappa}_{+S i} \theta^{j}-\text { h.c }\right)\left(u \gamma^{\prime m^{\prime}} u^{-1}\right)_{j}^{i}+\mathcal{O}(\theta \wedge \vartheta) .
\end{aligned}
$$

Again, the conditions $\delta x^{m}=0$ and $\theta^{i}=0$ imply two decomposition laws (up to cubic fermion terms)

$$
\begin{aligned}
\zeta^{\mu}(\sigma)= & \xi_{C}^{\mu}(\sigma)+\frac{1}{2}\left(\bar{\epsilon}_{i}(\sigma) \gamma^{\mu} \theta^{i}+\text { h.c. }\right)+\left(\frac{R}{\rho}\right)^{2}\left[\lambda_{(K)}^{\mu}+\frac{1}{2}\left(\bar{\eta}_{i} \gamma^{\mu} \vartheta^{i}+\text { h.c. }\right)\right] \\
& +\frac{1}{2}\left(\bar{\kappa}_{+Q i} \gamma^{\mu} \theta^{i}+\text { h.c. }\right)+\left(\frac{R}{\rho}\right)^{2}\left[\frac{1}{2}\left(\bar{\kappa}_{+S i} \gamma^{\mu} \vartheta^{i}+\text { h.c. }\right)\right] \\
& +\mathcal{O}(\theta \wedge \vartheta),
\end{aligned}
$$

and,

$$
\kappa_{+Q}^{i}=\epsilon^{i}(x)+\left(\frac{R}{\rho}\right)^{2} \lambda_{(K)}^{m} \gamma_{m} \vartheta^{i} .
$$

The remaining worldvolume fields (apart from the worldvolume vector which we ignore in this paper) are then $\rho(\sigma), z^{m^{\prime}}(\sigma)$ and $\vartheta(\sigma)$ and their transformation rules are the following (up to cubic fermion terms)

$$
\begin{aligned}
\delta \vartheta^{i}= & \hat{\xi}_{C}^{\mu}(\sigma) \partial_{\mu} \vartheta^{i}-\frac{1}{4} \Lambda_{M}(\sigma) \cdot \gamma \vartheta^{i}+\frac{1}{2} \Lambda_{D}(\sigma) \vartheta^{i}-\frac{1}{4} \vartheta^{j} \Lambda_{\mathrm{SO}(6)}^{I J}\left(\hat{\gamma}_{I J}^{\prime}\right)_{j}{ }^{i} \\
& -\frac{\beta_{+}}{R}\left(\frac{R}{\rho}\right)^{2} \lambda_{(K)}^{m} \gamma_{m} \vartheta^{i}-\frac{\beta_{+}}{R} \epsilon^{i}(\sigma)-\eta^{i},
\end{aligned}
$$




$$
\begin{aligned}
\delta \rho= & \hat{\xi}_{C}^{\mu}(\sigma) \partial_{\mu} \rho+\Lambda_{D}(\sigma) \rho-\left[\bar{\vartheta}_{i}\left(\epsilon^{i}+\frac{1}{2}\left(\frac{R}{\rho}\right)^{2} \lambda_{(K)}^{m} \gamma_{m} \vartheta^{i}\right)+\text { h.c }\right] \rho \\
& +\frac{1}{2}\left(\frac{R}{\rho}\right)^{2}\left[\bar{\eta}_{i} \gamma^{\mu} \vartheta^{i}+\bar{\vartheta}_{i} \gamma^{\mu} \frac{\beta_{+}}{R}\left(\epsilon^{i}(\sigma)+\left(\frac{R}{\rho}\right)^{2} \lambda_{(K)}^{m} \gamma_{m} \vartheta^{i}\right)+\text { h.c. }\right] \partial_{\mu} \rho, \\
\delta z^{m^{\prime}}= & \hat{\xi}_{C}^{\mu}(\sigma) \partial_{\mu} z^{m^{\prime}}-\xi^{m^{\prime}}(z) \\
& +\frac{\left(1-z^{2}\right)}{2}\left[\bar{\epsilon}_{i}(\sigma) \vartheta^{j}+\frac{1}{2}\left(\frac{R}{\rho}\right)^{2} \Lambda_{K}^{m} \bar{\vartheta}_{i} \gamma_{m} \vartheta^{j}+\text { h.c. }\right]\left(u \gamma^{\prime m^{\prime}} u^{-1}\right)_{j}^{i} \\
& +\frac{1}{2}\left(\frac{R}{\rho}\right)^{2}\left[\bar{\eta}_{i} \gamma^{\mu} \vartheta^{i}+\bar{\vartheta}_{i} \gamma^{\mu} \frac{\beta_{+}}{R}\left(\epsilon^{i}(\sigma)+\left(\frac{R}{\rho}\right)^{2} \Lambda_{K}^{m} \gamma_{m} \vartheta^{i}\right)+\text { h.c. }\right] \partial_{\mu} z^{m^{\prime}}
\end{aligned}
$$

where

$$
\hat{\xi}_{C}^{\mu}(\sigma) \equiv \xi_{C}^{\mu}(\sigma)+\left(\frac{R}{\rho}\right)^{2} \lambda_{(K)}^{\mu}
$$

\section{From $A d S_{5} \times S^{5}$ to Minkowski: the large $R$ limit}

We want to compare the resulting worldvolume transformations of the two backgrounds discussed in the previous section. Our aim is to establish a relation between the symmetries in $A d S_{5} \times S^{5}$ background and the Volkov-Akulov supersymmetries in the Minkowski background of [1]. In order to make an identification, we need to take a suitable large $R$ limit of the $A d S_{5} \times S^{5}$ background. We start out with a discussion of the proper limit.

To take this limit, it is convenient to change (background) spacetime coordinates. We define

$$
\rho=e^{r / R}, \quad z^{m^{\prime}}=\frac{\tilde{z}^{m^{\prime}}}{2 R}
$$

The metric (2.23) then becomes

$$
d s^{2}=e^{2 r / R} d x^{\mu} \eta_{\mu \nu} d x^{\nu}+e^{-2 r / R} d r^{2}+\frac{1}{\left(1+\tilde{z}^{2} /\left(4 R^{2}\right)\right)^{2}} d \tilde{z}^{2}
$$

which becomes Minkowski space in the limit $R \rightarrow \infty$. We also need to change variables in the algebra. The algebra we have used to derive the transformation rules in the previous sections relied on the conformal decomposition (appendix B.2) and the right variables for the algebra are the ones of the AdS decomposition (appendix B.1). Equation (B.19) gives the relation between the various decompositions and is to be used to obtain the right variables. In particular this means that we redefine variables

$$
\tilde{\vartheta}^{i}=R \vartheta^{i}, \quad \tilde{\eta}^{i}=R \eta^{i} \quad \text { and } \quad \tilde{\kappa}_{+S}^{i}=R \kappa_{+S}^{i}
$$

Applying these redefinitions and taking the large $R$ limit nicely reduces the $A d S_{5} \times S^{5}$ supervielbein and isometries, (2.25) and (2.26), to their Minkowski space equivalents, (2.18) and (2.19) (modulo the spacetime mixing requirement $\lambda_{(M)}^{m n^{\prime}}=\lambda_{(M)}^{4 n^{\prime}}=0$ ). We now apply 
this to the transformations of the worldvolume fields in the $A d S_{5} \times S^{5}$ background, (3.21), and take the limit $R \rightarrow \infty$ to obtain

$$
\begin{aligned}
\delta r= & \xi^{\mu} \partial_{\mu} r-\frac{1}{2}\left[\overline{\tilde{\vartheta}}_{i} \gamma^{\mu}\left(\tilde{\eta}^{i}-\beta_{+}\left(\epsilon^{i}-\frac{1}{2} \tilde{A}^{m S} \gamma_{m} \tilde{\vartheta}^{i}\right)\right)+\text { h.c. }\right] \partial_{\mu} r \\
& -\tilde{A}^{S}-\tilde{A}^{S n} x_{n}-\left[\overline{\tilde{\vartheta}}^{i}\left(\epsilon^{i}-\frac{1}{4} \tilde{A}^{m S} \gamma_{m} \tilde{\vartheta}^{i}\right)+\text { h.c. }\right], \\
\delta \tilde{z}^{m^{\prime}}= & \xi^{\mu} \partial_{\mu} \tilde{z}^{m^{\prime}}-\frac{1}{2}\left[\overline{\tilde{\vartheta}}_{i} \gamma^{\mu}\left(\tilde{\eta}^{i}-\beta_{+}\left(\epsilon^{i}-\frac{1}{2} \tilde{A}^{m S} \gamma_{m} \tilde{\vartheta}^{i}\right)\right)+\text { h.c. }\right] \partial_{\mu} \tilde{z}^{m^{\prime}} \\
& -\tilde{\xi}^{m^{\prime}}-\left[\left(\bar{\epsilon}_{i}-\frac{1}{4} \tilde{A}^{m S} \overline{\tilde{\vartheta}}_{i} \gamma_{m}\right) \tilde{\vartheta}^{j}\left(\gamma^{\prime m^{\prime}}\right)_{j}{ }^{i}+\text { h.c. }\right], \\
\delta \tilde{\vartheta}^{i}= & \xi^{\mu} \partial_{\mu} \tilde{\vartheta}^{i}-\frac{1}{4} A_{(M)}^{m n} \gamma_{m n} \tilde{\vartheta}^{i} \\
& -\frac{1}{4} \Lambda_{\mathrm{SO}(6)}^{I J}\left(\hat{\gamma}_{I J}^{\prime}\right)_{j}{ }^{i} \tilde{\vartheta}^{j}-\left[\tilde{\eta}^{i}+\beta_{+}\left(\epsilon^{i}-\frac{1}{2} \tilde{A}^{m S} \gamma_{m} \tilde{\vartheta}^{i}\right)\right]
\end{aligned}
$$

where $\xi^{\mu}=\tilde{A}^{\mu}+r \tilde{A}^{\mu S}+\tilde{A}^{\mu n} x_{n}$.

Making the identifications

$$
\begin{array}{llrl}
x_{\text {Mink }}^{5} & =r, & x_{\text {Mink }}^{m^{\prime}}=\tilde{z}^{m^{\prime}}, & \vartheta_{\text {Mink }}^{i}=\tilde{\vartheta}^{i} \\
\lambda_{\text {Mink }}^{m 4}=\tilde{A}^{m S}, & \eta_{\text {Mink }}^{i}=\tilde{\eta}^{i}, & \lambda_{(M), \text { Mink }}^{m n}=\tilde{A}_{(M)}^{m n},
\end{array}
$$

and

$$
\xi_{\text {Mink }}^{4}=\tilde{A}^{S}+\tilde{A}^{S m} x_{m},
$$

where the subscript Mink refers to the quantities in (3.17), we can compare (4.4) with (3.17) and we find an exact match between the worldvolume transformation rules.

However, there seems to be no way to link Minkowski background symmetries to the $A d S_{5} \times S^{5}$ symmetries without introducing a length scale, not at all a surprising result. The reason for this is quite simple and can be found by looking at the conformal algebra. The algebra corresponding to our $A d S_{5} \times S^{5}$ space was given in (B.17). We are interested in the anti-commutators of the fermionic generators which we repeat here for convenience

$$
\begin{aligned}
& \left\{Q_{\alpha}{ }^{i}, \bar{Q}_{j}{ }^{\beta}\right\}=\delta_{j}{ }^{i}\left(\gamma^{a}\right)_{\alpha}{ }^{\beta} P_{a}, \quad\left\{S_{\alpha}{ }^{i}, \bar{S}_{j}{ }^{\beta}\right\}=\delta_{j}{ }^{i}\left(\gamma^{a}\right)_{\alpha}{ }^{\beta} K_{a}, \\
& \left\{Q_{\alpha}{ }^{i}, \bar{S}_{j}{ }^{\beta}\right\}=\delta_{j}{ }^{i} \delta_{\alpha}{ }^{\beta} D+\delta_{j}{ }^{i}\left(\gamma^{a b}\right)_{\alpha}{ }^{\beta} M_{a b}-2 \delta_{\alpha}{ }^{\beta} U_{j}{ }^{i} .
\end{aligned}
$$

Before we take the limit $R \rightarrow \infty$, we need to write these anticommutators in the notation of the AdS decomposition. Using the relations in section B.2 we find

$$
\begin{aligned}
& \left\{\left(\mathcal{P}_{Q} \tilde{\mathcal{Q}}\right)_{\hat{\alpha}}^{i},\left(\overline{\mathcal{P}_{Q} \tilde{\mathcal{Q}}}\right)_{j}^{\hat{\beta}}\right\}=-\frac{1}{2} \delta_{j}{ }^{i}\left(\mathcal{P}_{Q} \hat{\gamma}^{a T}\right)_{\hat{\alpha}}^{\hat{\beta}}\left(\tilde{P}_{a}+\frac{2}{R} \tilde{M}_{a S}\right), \\
& \left\{\left(\mathcal{P}_{S} \tilde{\mathcal{Q}}\right)_{\hat{\alpha}}^{i},\left(\overline{\mathcal{P}_{S} \tilde{\mathcal{Q}}}\right)_{j}^{\hat{\beta}}\right\}=-\frac{1}{2} \delta_{j}{ }^{i}\left(\mathcal{P}_{S} \hat{\gamma}^{a T}\right)_{\hat{\alpha}}^{\hat{\beta}}\left(\tilde{P}_{a}-\frac{2}{R} \tilde{M}_{a S}\right), \\
& \left\{\left(\mathcal{P}_{Q} \tilde{\mathcal{Q}}\right)_{\hat{\alpha}}^{i},\left(\overline{\mathcal{P}_{S} \tilde{\mathcal{Q}}}\right)_{j}^{\hat{\beta}}\right\}=-\frac{1}{2} \delta_{j}{ }^{i}\left(\mathcal{P}_{Q}\right)_{\hat{\alpha}}^{\hat{\beta}} \tilde{P}_{S}+\frac{1}{2 R} \delta_{j}{ }^{i}\left(\mathcal{P}_{Q} \hat{\gamma}^{a b}\right)_{\hat{\alpha}}^{\hat{\beta}} M_{a b}-\frac{1}{R}\left(\mathcal{P}_{Q}\right)_{\hat{\alpha}}{ }^{\hat{\beta}} U_{j}{ }^{i} .
\end{aligned}
$$


From these commutation relations it is clear that in the limit $R \rightarrow \infty$ the right hand side of the first two commutators reduces to a translation. In other words, the distinction between the operator $P_{a}$ and the operator $K_{a}$ disappears. The conformal structure is an $\mathcal{O}\left(\frac{1}{R}\right)$-effect, and requires a length scale used for separation to work.

In light of this it is also clear why the major difference of the Volkov-Akulov supersymmetries of [1] and conformal supersymmetry rests in the Volkov-Akulov supersymmetries anti-commuting into translations. There simply is no length scale from the background available to make the distinction between translations and special conformal transformations. Let us look at the relation with the results from [1] a bit closer. In order to really compare with [1], we should write our transformations in a form that looks like (only considering the fermionic symmetries now)

$$
\begin{aligned}
\delta \phi^{I} & \sim\left(\bar{\lambda}_{i} \Gamma^{\mu} \epsilon^{2 i}+\bar{\lambda}_{i} \Gamma^{\mu} \beta_{+} \epsilon^{1 i}\right) \partial_{\mu} \phi^{I}, \\
\delta \lambda^{i} & \sim \epsilon^{2 i}+\beta_{+} \epsilon^{1 i} .
\end{aligned}
$$

Looking at the transformations (4.4), we find

$$
\epsilon^{1}=\epsilon^{i}-\frac{1}{2} \lambda^{n 4} \gamma_{n} \vartheta^{i}, \quad \epsilon^{2}=\eta^{i}+2 \epsilon^{i}-\frac{1}{2} \lambda^{n 4} \gamma_{n} \vartheta^{i} .
$$

In order to make the appearance of the Volkov-Akulov symmetry apparent, we define the parameters

$$
\begin{aligned}
& \varepsilon=\epsilon^{1}=\epsilon^{i}-\frac{1}{2} \lambda^{n 4} \gamma_{n} \vartheta^{i}, \\
& \zeta=\epsilon^{2}-\epsilon^{1}=\eta^{i}+\epsilon^{i},
\end{aligned}
$$

suggesting that the generators for supersymmetry and Volkov-Akulov symmetry will be

$$
\left(Q_{\mathrm{SUSY}}\right)_{\hat{\alpha}}^{i}=\left(\mathcal{P}_{Q} \tilde{\mathcal{Q}}\right)_{\hat{\alpha}}^{i}, \quad\left(Q_{\mathrm{VA}}\right)_{\hat{\alpha}}^{i}=\left(\mathcal{P}_{Q} \tilde{\mathcal{Q}}\right)_{\hat{\alpha}}^{i}+\left(\mathcal{P}_{S} \tilde{\mathcal{Q}}\right)_{\hat{\alpha}}^{i}
$$

The corresponding algebra becomes

$$
\begin{aligned}
\left\{\left(Q_{\mathrm{SUSY}}\right)_{\hat{\alpha}}{ }^{i},\left(\overline{Q_{\mathrm{SUSY}}}\right)_{j}^{\hat{\beta}}\right\} & =-\frac{1}{2} \delta_{j}{ }^{i}\left(\mathcal{P}_{Q} \hat{\gamma}^{a T}\right)_{\hat{\alpha}}^{\hat{\beta}} \tilde{P}_{a} \\
\left\{\left(Q_{\mathrm{VA}}\right)_{\hat{\alpha}}^{i},\left(\overline{Q_{\mathrm{VA}}}\right)_{j}^{\hat{\beta}}\right\} & =-\frac{1}{2} \delta_{j}{ }^{i}\left(\hat{\gamma}^{a T}\right)_{\hat{\alpha}}^{\hat{\beta}} \tilde{P}_{a}-\frac{1}{2} \delta_{j}{ }^{i}(\delta)_{\hat{\alpha}}^{\hat{\beta}} \tilde{P}_{S}-\frac{1}{R}(\delta)_{\hat{\alpha}}^{\hat{\beta}} U_{j}{ }^{i} \\
\left\{\left(Q_{\mathrm{SUSY}}\right)_{\hat{\alpha}}{ }^{i},\left(\overline{Q_{\mathrm{VA}}}\right)_{j}^{\hat{\beta}}\right\} & =-\frac{1}{2} \delta_{j}{ }^{i}\left(\mathcal{P}_{Q} \hat{\gamma}^{a T}\right)_{\hat{\alpha}}^{\hat{\beta}} \tilde{P}_{a}-\frac{1}{2} \delta_{j}{ }^{i}\left(\mathcal{P}_{Q}\right)_{\hat{\alpha}}^{\hat{\beta}} \tilde{P}_{S}-\frac{1}{R}\left(\mathcal{P}_{Q}\right)_{\hat{\alpha}}^{\hat{\beta}} U_{j}{ }^{i}
\end{aligned}
$$

where we clearly see the appearance of translations and shift-symmetries of the scalar fields in the anti-commutators of the Volkov-Akulov-symmetry.

\section{Conclusions}

We compared the worldvolume transformation rules of a D3-brane embedded in a Minkowski background with those of a D3-brane embedded in an $A d S_{5} \times S^{5}$ background. 
We obtained a relation between the special supersymmetry transformations induced by the $A d S_{5} \times S^{5}$ background and the Volkov-Akulov symmetries related to the Minkowski background. In order to relate one to the other, one needs to introduce a length scale, a result that is reaffirmed by looking at the algebra. The existence of a length scale in the algebra associated to the $A d S_{5} \times S^{5}$ background allows for the distinction between translations and special conformal translations as an $\mathcal{O}\left(\frac{1}{R}\right)$-effect. When this effect is very small (at large $R$ ) this distinction disappears, and it is therefore no surprise that in a Minkowski background one only finds supersymmetry transformations that anti-commute into translations and shift-symmetries (i.e. the 16 supersymmetries +16 Volkov-Akulov symmetries of [1]).

The question remains then whether we can construct higher derivative invariants coupled to supergravity in the $D=4, \mathcal{N}=4$ setting with VA-type symmetries. We will provide a tentative scheme for constructing these higher derivative invariants. Having established a relation between the conformal symmetry inherited by the $A d S_{5} \times S^{5}$ background and the Volkov-Akulov symmetry due to the Minkowski background, we can use this relation as a tool for the construction of higher derivative invariants. The idea is to perform a construction of higher derivative invariants using superconformal methods in the theory of the brane embedded in $A d S_{5} \times S^{5}$, followed by making the redefinitions (4.1) and (4.3), and then taking the limit necessary to obtain the Minkowski background. Our gauge choice to fix $\kappa$-symmetry is special in the sense that it has an easy limit to obtain the worldvolume theory of a D3-brane in a Minkowski background. However, for the practical application of superconformal methods it is less convenient since it does not have a simple, linearly realised form for the supersymmetries. How to fix the $\kappa$-symmetry gauge in the $A d S_{5} \times S^{5}$ background in the most natural way is still an open problem [18]. The gauge choice we made in this paper, however, makes the relation with the transformation rules in the Minkowski background clear, and should be related to this unknown gauge choice by field redefinitions. If we can find such a gauge choice to simplify the construction of higher derivative invariants, we can modify the scheme by starting from this case with (as of yet) unknown $\kappa$-symmetry gauge to construct higher derivative invariants using superconformal methods. Once these are constructed field redefinitions will transform these higher derivative invariants to the gauge used in this paper, the $Q$-gauge. It is then only a matter of taking the large $R$-limit to obtain higher derivative invariants in the desired $D=4, \mathcal{N}=4$ setting with VA-type symmetries.

\section{Acknowledgments}

We thank A. Van Proeyen for useful conversations. The work of B.V.P was supported in part by the FWO - Vlaanderen, Project No. G.0651.11, and in part by the Interuniversity Attraction Poles Programme initiated by the Belgian Science Policy (P7/37).

\section{A Clifford algebras}

We follow the notation and conventions of [7]. For ease of use, we collect here a few of the Clifford algebra's and their realisations. For the two background cases we need Clifford 
matrices tailored to their needs. We start of by constructing the algebras needed for the $A d S_{5} \times S^{5}$ case $(\mathrm{SO}(2,4)$ and $\mathrm{SO}(6))$ and conclude with a compatible $\mathrm{SO}(1,9)$ construction for the Minkowski background.

\section{A.1 The $\mathrm{SO}(2,4)$ Clifford algebra}

We extend the $\mathrm{SO}(1,3)$ Clifford matrices by two more matrices as follows

$$
\hat{\Gamma}_{a}=\gamma_{a} \otimes \sigma_{1}, \quad \hat{\Gamma}_{S}=\gamma_{4} \otimes \sigma_{1}, \quad \hat{\Gamma}_{T}=I_{4} \otimes\left(-i \sigma_{2}\right)
$$

The $\gamma_{a}$ are the $\mathrm{SO}(1,3)$ gamma matrices and $\gamma_{4}=-i \gamma_{0} \gamma_{1} \gamma_{2} \gamma_{3}$. We define

$$
\hat{\Gamma}_{*}=-i \hat{\Gamma}_{0} \hat{\Gamma}_{1} \ldots \hat{\Gamma}_{S} \hat{\Gamma}_{T}=I \otimes \sigma_{3} .
$$

Since we are in 6 dimensions the minimal spinor is a Weyl spinor, the conformal spinor in 4 dimensions. We will restrict to righthanded chiral spinors, $\hat{\Gamma}_{*} \lambda=-\lambda$. We restrict $\hat{\Gamma}_{a b}$ to the righthanded chiral subspace $^{1}(\hat{\Gamma} \rightarrow \hat{\gamma})$

$$
\hat{\gamma}_{a b}=\gamma_{a b}, \quad \hat{\gamma}_{a S}=\gamma_{a} \gamma_{5}, \quad \hat{\gamma}_{a T}=-\gamma_{a}, \quad \hat{\gamma}_{S T}=-\gamma_{5} .
$$

These matrices satisfy the relations

$$
\left(\hat{\gamma}_{\hat{a} \hat{b}}\right)_{\hat{\alpha}}^{\hat{\beta}}\left(\hat{\gamma}^{\hat{a} \hat{b}}\right)_{\hat{\gamma}}^{\hat{\delta}}=2 \delta_{\hat{\alpha}}^{\hat{\beta}} \delta_{\hat{\gamma}}^{\hat{\delta}}-8 \delta_{\hat{\alpha}}^{\hat{\delta}} \delta_{\hat{\gamma}}^{\hat{\beta}}, \quad\left(\hat{\gamma}_{\hat{a} \hat{b}}\right)_{\hat{\alpha}}^{\hat{\beta}}\left(\hat{\gamma}^{\hat{c} \hat{d}}\right)_{\hat{\beta}}^{\hat{\alpha}}=-8 \delta_{[\hat{a}}^{\hat{c}} \delta_{\hat{b}]}^{\hat{d}},
$$

where for this section $\hat{\alpha}=1, \ldots 8$ and $\hat{a}=\{a, S, T\}$.

\section{A.2 The $\mathrm{SO}(6)$ Clifford algebra}

We extend the $\mathrm{SO}(5)$ Clifford matrices by one more matrix

$$
\hat{\Gamma}_{a^{\prime}}^{\prime}=\gamma_{a^{\prime}}^{\prime} \otimes \sigma_{2}, \quad \hat{\Gamma}_{S^{\prime}}^{\prime}=\gamma_{9}^{\prime} \otimes \sigma_{2}, \quad \hat{\Gamma}_{T^{\prime}}^{\prime}=I_{4} \otimes \sigma_{1},
$$

where $\gamma_{a^{\prime}}^{\prime}$ are the $\mathrm{SO}(4)$ gamma matrices and $\gamma_{9}^{\prime}$ is given by $\gamma_{9}^{\prime}=-\gamma_{5}^{\prime} \gamma_{6}^{\prime} \gamma_{7}^{\prime} \gamma_{8}^{\prime}$. We define

$$
\hat{\Gamma}_{*}^{\prime}=-i \hat{\Gamma}_{5}^{\prime} \hat{\Gamma}_{6}^{\prime} \ldots \hat{\Gamma}_{S^{\prime}}^{\prime} \hat{\Gamma}_{T^{\prime}}^{\prime}=I \otimes \sigma_{3}
$$

Like before we will restrict to righthanded chiral spinors, and identify

$$
\hat{\gamma}_{a^{\prime} b^{\prime}}^{\prime}=\gamma_{a^{\prime} b^{\prime}}^{\prime}, \quad \hat{\gamma}_{a^{\prime} S^{\prime}}^{\prime}=\gamma_{a^{\prime}}^{\prime} \gamma_{9}^{\prime}, \quad \hat{\gamma}_{a^{\prime} T^{\prime}}^{\prime}=i \gamma_{a^{\prime}}^{\prime}, \quad \hat{\gamma}_{S^{\prime} T^{\prime}}^{\prime}=i \gamma_{9}^{\prime} .
$$

These matrices satisfy a similar relation as (A.4)

\footnotetext{
${ }^{1}$ This means that $\hat{\gamma}_{\tilde{a} \tilde{b}}=\hat{\Gamma}_{\tilde{a} \tilde{b}} \frac{1}{2}\left(1-\hat{\Gamma}_{*}\right)$.
} 


\section{A.3 The $\mathrm{SO}(1,9)$ Clifford algebra}

We will use a decomposition of 10-dimensional $\gamma$-matrices $\hat{\Gamma}_{M}^{(10 \mathrm{D})}$ into the $\mathrm{SO}(1,4)$ and $\mathrm{SO}(5)$ matrices as follows

$$
\hat{\Gamma}_{\tilde{m}}^{(10 \mathrm{D})}=\gamma_{\tilde{m}} \otimes I_{4} \otimes \sigma_{1}, \quad \hat{\Gamma}_{m^{\prime}}^{(10 \mathrm{D})}=I_{4} \otimes \gamma_{m^{\prime}}^{\prime} \otimes \sigma_{2} .
$$

We define

$$
\hat{\Gamma}_{*}^{(10 \mathrm{D})}=-\hat{\Gamma}_{0}^{(10 \mathrm{D})} \cdots \hat{\Gamma}_{9}^{(10 \mathrm{D})}=-I_{4} \otimes I_{4} \otimes \sigma_{3}
$$

We can write 10-dimensional spinors in this decomposition as

$$
\Psi_{(10 \mathrm{D})}=\psi \otimes \psi^{\prime} \otimes\left(\begin{array}{l}
a \\
b
\end{array}\right)
$$

however, the type IIB chirality condition $\frac{1}{2}\left(1+\hat{\Gamma}_{*}^{(10 \mathrm{D})}\right) \Psi_{(10 \mathrm{D})}=0$ implies that

$$
0=\frac{1}{2}\left(1+\hat{\Gamma}_{*}^{(10 \mathrm{D})}\right) \Psi_{(10 \mathrm{D})}=\psi \otimes \psi^{\prime} \otimes\left(\begin{array}{l}
0 \\
b
\end{array}\right) \quad \rightarrow \quad b=0 .
$$

Our 10-dimensional chiral spinor is then

$$
\Psi_{\alpha}^{i}=\psi_{\alpha} \otimes \psi^{\prime i} \otimes\left(\begin{array}{l}
1 \\
0
\end{array}\right),
$$

where we reabsorbed the constant $a$ into the 4-dimensional spinors. By doing this restriction to the right handed chiral subspace we can again define

$$
\Gamma_{\hat{m} \hat{n}}=\hat{\Gamma}_{\hat{m} \hat{n}}^{(10 \mathrm{D})} \frac{1}{2}\left(1+\hat{\Gamma}_{*}^{(10 \mathrm{D})}\right),
$$

and identify

$$
\Gamma_{\tilde{m} \tilde{n}}=\gamma_{\tilde{m} \tilde{n}} \otimes I_{4}, \quad \Gamma_{\tilde{m}^{\prime} \tilde{n}^{\prime}}=I_{4} \otimes \gamma_{\tilde{m}^{\prime} \tilde{n}^{\prime}}^{\prime}, \quad \Gamma_{\tilde{m} \tilde{n}^{\prime}}=\gamma_{\tilde{m}} \otimes \gamma_{\tilde{n}^{\prime}}^{\prime}
$$

\section{B The SU(2, 2|4) algebra in various forms}

The $\mathrm{SU}(2,2 \mid 4)$ algebra is

$$
\begin{aligned}
& {\left[V_{\hat{\alpha}}{ }^{\hat{\beta}}, V_{\hat{\gamma}}{ }^{\hat{\delta}}\right]=\delta_{\hat{\gamma}}{ }^{\hat{\beta}} V_{\hat{\alpha}}{ }^{\hat{\delta}}-\delta_{\hat{\alpha}}{ }^{\hat{\delta}} V_{\hat{\gamma}}{ }^{\hat{\beta}}, \quad\left[U_{i}{ }^{j}, U_{k}{ }^{l}\right]=\delta_{i}{ }^{l} U_{k}{ }^{j}-\delta_{k}{ }^{j} U_{i}{ }^{l}} \\
& {\left[V_{\hat{\alpha}}{ }^{\hat{\beta}}, \mathcal{Q}_{\hat{\gamma}}{ }^{i}\right]=\delta_{\hat{\gamma}}{ }^{\hat{\beta}} \mathcal{Q}_{\hat{\alpha}}{ }^{i}-\frac{1}{4} \delta_{\hat{\alpha}}{ }^{\hat{\beta}} \mathcal{Q}_{\hat{\gamma}}{ }^{i}, \quad\left[U_{i}{ }^{j}, \mathcal{Q}_{\hat{\alpha}}{ }^{k}\right]=\delta_{i}{ }^{k} \mathcal{Q}_{\hat{\alpha}}{ }^{j}-\frac{1}{4} \delta_{i}{ }^{j} \mathcal{Q}_{\hat{\alpha}}^{k} \text {, }} \\
& \left\{\mathcal{Q}_{\hat{\alpha}}{ }^{i}, \overline{\mathcal{Q}}_{j}{ }^{\hat{\beta}}\right\}=\delta_{j}{ }^{i} V_{\hat{\alpha}}{ }^{\hat{\beta}}-\delta_{\hat{\alpha}}{ }^{\hat{\beta}} U_{j}{ }^{i},
\end{aligned}
$$

with all other commutators vanishing. The index $\hat{\alpha}$ runs over the values $1, \ldots 4$ in this section. 
We will relate the fundamental representation of $\mathrm{SU}(2,2)$ to the spinor of $\mathrm{SO}(2,4)$. We rotate the generators $V_{\hat{\alpha}}^{\hat{\beta}}$ to $\hat{M}_{\hat{m} \hat{n}}$ by means of the $\hat{\gamma}_{\hat{a} \hat{b}}$ matrices given in (A.3)

$$
V_{\hat{\alpha}}^{\hat{\beta}}=\frac{1}{2}\left(\hat{\gamma}^{\hat{a} \hat{b}}\right)_{\hat{\alpha}}^{\hat{\beta}} \hat{M}_{\hat{a} \hat{b}}, \quad \hat{M}_{\hat{a} \hat{b}}=-\frac{1}{4}\left(\hat{\gamma}_{\hat{a} \hat{b}}\right)_{\hat{\alpha}}^{\hat{\beta}} V_{\hat{\beta}}^{\hat{\alpha}} .
$$

This is consistent by the fact that the $V_{\hat{\alpha}}^{\hat{\beta}}$ are traceless and (A.4). The other bosonic subalgebra, generated by $U$ will be considered as an internal group (an $R$-symmetry) for the remainder of this section. The conjugate spinor charge $\overline{\mathcal{Q}}_{i}{ }^{\hat{\alpha}}$ is defined as the fourdimensional Dirac conjugate spinor,

$$
\overline{\mathcal{Q}}_{i}^{\hat{\alpha}}=i\left[\left(\mathcal{Q}^{i}\right)^{\dagger} \gamma^{0}\right]^{\hat{\alpha}}
$$

With this isomorphism realised, we have a superalgebra in terms of the generators

$$
\mathbf{T}_{\Lambda}: \quad \hat{M}_{\hat{a} \hat{b}}, \quad U_{i}^{j}, \quad \mathcal{Q}_{\hat{\alpha}}^{i} \quad \text { and } \quad \overline{\mathcal{Q}}^{i}{ }^{\hat{\alpha}}
$$

The super spacetime part of the algebra now gets the universal form

$$
\begin{aligned}
{\left[\hat{M}_{\hat{a} \hat{b}}, \hat{M}_{\hat{c} \hat{d}}\right] } & =\hat{\eta}_{\hat{a}[\hat{c}} \hat{M}_{\hat{d}] \hat{b}}-\hat{\eta}_{\hat{b}[\hat{c}} \hat{M}_{\hat{d}] \hat{a}}, \\
{\left[\hat{M}_{\hat{a} \hat{b}}, \mathcal{Q}_{\hat{\alpha}}{ }^{i}\right] } & =-\frac{1}{4}\left(\hat{\gamma}_{\hat{a} \hat{b}}\right)_{\hat{\alpha}}^{\hat{\beta}} \mathcal{Q}_{\hat{\beta}}{ }^{i}, \\
\left\{\mathcal{Q}_{\hat{\alpha}}{ }^{i}, \mathcal{Q}_{j}{ }^{\hat{\beta}}\right\} & \sim \delta_{j}{ }^{i}\left(\hat{\gamma}^{\hat{a} \hat{b}}\right)_{\hat{\alpha}}{ }^{\hat{\beta}} \hat{M}_{\hat{a} \hat{b}}+\delta_{\hat{\alpha}}{ }^{\hat{\beta}} U_{i}{ }^{j},
\end{aligned}
$$

and there is the internal part which involves the generators $U_{i}{ }^{j}$, which also rotate the supercharges. The metric $\hat{\eta}=\operatorname{diag}(-++++-)$ is the $(2,4)$ flat metric and the indices $\hat{a}=\{0,1,2,3, S, T\}$ where 0 and $T$ are timelike directions. Remark that we chose all generators in this formula to be dimensionless. In general we define a $\mathbf{G}$ valued object $A$ as

$$
A=A^{\Lambda} \mathbf{T}_{\Lambda}
$$

For the superalgebra above we have

$$
A=\hat{A}^{\hat{a} \hat{b}} \hat{M}_{\hat{a} \hat{b}}+\hat{A}_{i}^{j} U_{j}^{i}+\overline{\hat{A}}_{i}^{\hat{\alpha}} \mathcal{Q}_{\hat{\alpha}}^{i}+\overline{\mathcal{Q}}_{i}{ }^{\hat{\alpha}} \hat{A}_{\hat{\alpha}}^{i}
$$

where these objects can be viewed as matrices. Note that $\overline{\mathcal{Q}}_{i}{ }^{\hat{\alpha}}$ does not act on $\hat{A}_{\hat{\alpha}}{ }^{i}$ in this notation.

We want to derive the generators of the AdS algebra and the conformal algebra in their more familiar form. Starting from the generic form of the conformal superalgebra in the $\mathrm{SO}(2,4)$ basis $(\mathrm{B} .5)$, we will first decompose it into a form which is appropriate to the $A d S_{5}$ spacetime isometry algebra and then into a form which is appropriate for the conformal isometries in 4 dimensions. We call these the AdS decomposition and the conformal decomposition, respectively. We will also discuss how quantities in these decompositions are related. 


\section{B.1 The AdS decomposition}

The $A d S_{5}$ space is a 5-dimensional manifold with structure group $\mathrm{SO}(2,4)$, in order to obtain this from the algebra we split the generators into $\mathrm{SO}(1,4)$ generators $\tilde{M}_{\tilde{m} \tilde{n}}$ and the remaining generators $\tilde{P}_{\tilde{m}}$, defined through

$$
\tilde{P}_{\tilde{m}}=\frac{2}{R} \hat{M}_{\tilde{m} T}, \quad \tilde{M}_{\tilde{m} \tilde{n}}=\hat{M}_{\tilde{m} \tilde{n}}
$$

where we have introduced the constant $R$, which has dimensions of a length to give the translations $\tilde{P}_{\tilde{m}}$ the canonical dimensions of $L^{-1}$. It will be associated with the radius of curvature of the AdS space. The $S$-direction will be associated with the AdS bulk direction.

The supercharges $\mathcal{Q}_{\hat{\alpha}}{ }^{i}$ are rescaled to have dimensions $L^{-1 / 2}$,

$$
\tilde{\mathcal{Q}}_{\hat{\alpha}}^{i}=R^{-1 / 2} \mathcal{Q}_{\hat{\alpha}}^{i}
$$

This yields a superalgebra of the form

$$
\begin{array}{rlrl}
{\left[\tilde{M}_{\tilde{a} \tilde{b}}, \tilde{M}_{\tilde{c} \tilde{d}}\right]} & =\tilde{\eta}_{\tilde{a}[\tilde{c}} \tilde{M}_{\tilde{d}] \tilde{b}}-\tilde{\eta}_{\tilde{b}[\tilde{c}} \tilde{M}_{\tilde{d}] \tilde{a}}, & \\
{\left[\tilde{P}_{\tilde{a}}, \tilde{M}_{\tilde{b} \tilde{c}}\right]} & =\tilde{\eta}_{\tilde{a}[\tilde{b}} \tilde{P}_{\tilde{c}]}, & {\left[\tilde{P}_{\tilde{a}}, \tilde{P}_{\tilde{b}}\right]=\frac{2}{R^{2}} \tilde{M}_{\tilde{a} \tilde{b}},} \\
{\left[\tilde{M}_{\tilde{a} \tilde{b}}, \tilde{\mathcal{Q}}_{\hat{\alpha}}{ }^{i}\right]} & =-\frac{1}{4}\left(\hat{\gamma}_{\tilde{a} \tilde{b}}\right)_{\hat{\alpha}}^{\hat{\beta}} \tilde{\mathcal{Q}}_{\hat{\beta}}{ }^{i}, & {\left[\tilde{P}_{\tilde{a}}, \tilde{\mathcal{Q}}_{\hat{\alpha}}{ }^{i}\right]=\frac{2}{R}\left(\hat{\gamma}_{\tilde{a} T}\right)_{\hat{\alpha}}{ }^{\hat{\beta}} \tilde{\mathcal{Q}}_{\hat{\beta}}{ }^{i}} \\
\left\{\tilde{\mathcal{Q}}_{\hat{\alpha}}{ }^{i}, \tilde{\mathcal{Q}}_{j}{ }^{\hat{\beta}}\right\} & \sim \delta_{j}{ }^{i}\left(\hat{\gamma}^{\tilde{a} T}\right)_{\hat{\alpha}}{ }^{\hat{\beta}} \tilde{P}_{\tilde{a}}+\frac{1}{R} \delta_{j}{ }^{i}\left(\hat{\gamma}^{\tilde{a} \tilde{b}}\right)_{\hat{\alpha}} \hat{\beta}^{\hat{\beta}} \tilde{M}_{\tilde{a} \tilde{b}}+\frac{1}{R} \delta_{\hat{\alpha}}{ }^{\hat{\beta}} U_{i}{ }^{j},
\end{array}
$$

where $\tilde{\eta}=\operatorname{diag}(-++++)$ is the flat metric with signature $(1,4)$.

It is interesting to note that this algebra contains the dimensionful constant $R$, which can not be scaled away if we want the translations to have natural dimension of a mass.

For the AdS superalgebra, we have the decomposition of a G-valued object

$$
A=\tilde{A}^{\tilde{a}} \tilde{P}_{\tilde{a}}+\tilde{A}^{\tilde{a} \tilde{b}} \tilde{M}_{\tilde{a} \tilde{b}}+\tilde{A}_{i}{ }^{j} U_{j}{ }^{i}+\overline{\tilde{A}}_{i}{ }^{\alpha} \tilde{\mathcal{Q}}_{\hat{\alpha}}{ }^{i}+\overline{\tilde{\mathcal{Q}}}_{i}{ }^{\hat{\alpha}} \tilde{A}_{\hat{\alpha}}{ }^{i} .
$$

From this we infer that

$$
\tilde{A}^{\tilde{a}}=R \hat{A}^{\tilde{a} T}, \quad \tilde{A}^{\tilde{a} \tilde{b}}=\hat{A}^{\tilde{a} \tilde{b}}, \quad \tilde{A}_{i}{ }^{j}=\hat{A}_{i}{ }^{j}, \quad \tilde{A}_{\hat{\alpha}}{ }^{i}=R^{1 / 2} \hat{A}_{\hat{\alpha}}{ }^{i} .
$$

\section{B.2 The conformal decomposition}

We now turn to the conformal decomposition of the superalgebra. We obtain the conformal transformations (generators) in 4 dimensions, i.e. translations $\left(P_{a}\right)$, Lorentz transformations $\left(M_{a b}\right)$, dilatations $(D)$ and special conformal transformations $\left(K_{a}\right)$, which also form the algebra $\mathrm{SO}(2,4)$, by

$$
\begin{aligned}
P_{a} & =\frac{2}{R}\left(\hat{M}_{a T}+\hat{M}_{a S}\right)=\tilde{P}_{a}+\frac{2}{R} \tilde{M}_{a S}, & {\left[P_{a}\right] } & =L^{-1}, \\
M_{a b} & =\hat{M}_{a b}=\tilde{M}_{a b}, & {\left[M_{a b}\right] } & =L^{0}, \\
D & =2 \hat{M}_{T S}=-R \tilde{P}_{S}, & {[D] } & =L^{0}, \\
K_{a} & =2 R\left(\hat{M}_{a T}-\hat{M}_{a S}\right)=R^{2} \tilde{P}_{a}-2 R \tilde{M}_{a S}, & {\left[K_{a}\right] } & =L,
\end{aligned}
$$


where we indicated the natural length dimensions $L$. The tangent directions have now been split as $\hat{a}=\{\tilde{a}, T\}=\{a, S, T\}$ and we introduce the 4-dimensional Minkowski metric $\eta_{a b}=$ $\operatorname{diag}(-,+,+,+)$. It is natural to split the supercharge $\mathcal{Q}_{\hat{\alpha}}{ }^{i}$ into two Lorentz supercharges, the supersymmetry $Q_{\alpha}{ }^{i}$ and the conformal supersymmetry $S_{\alpha}{ }^{i}$. One way to distinguish between the two is that they transform with opposite weight under the dilatations. Consider the commutator

$$
\left[D, \mathcal{Q}_{\hat{\alpha}}^{i}\right]=-\frac{1}{2} \hat{\gamma}_{T S} \mathcal{Q}_{\hat{\alpha}}{ }^{i}
$$

Since $\hat{\gamma}_{T S}^{2}=1$ and $\operatorname{Tr} \hat{\gamma}_{T S}=0$, we can define the projection operators

$$
\mathcal{P}_{Q, S}=\frac{1}{2}\left(1 \pm \hat{\gamma}_{S T}\right)
$$

We note that $\hat{\gamma}_{S T}$ commutes with $\hat{\gamma}_{a b}$ and therefore preserves the 4-dimensional Lorentz spinors as desired. This leads us to the following identification

$$
\text { supersymmetry: } \quad Q_{\alpha}{ }^{i}=\sqrt{2} R^{-1 / 2} \mathcal{P}_{Q} \mathcal{Q}_{\hat{\alpha}}{ }^{i}=\sqrt{2} \mathcal{P}_{Q} \tilde{\mathcal{Q}}_{\hat{\alpha}}{ }^{i}, \quad\left[Q_{\alpha}{ }^{i}\right]=L^{-1 / 2}
$$

special supersymmetry: $\quad S_{\alpha}{ }^{i}=\sqrt{2} R^{1 / 2} \mathcal{P}_{S} \mathcal{Q}_{\hat{\alpha}}{ }^{i}=\sqrt{2} R \mathcal{P}_{S} \tilde{\mathcal{Q}}_{\hat{\alpha}}{ }^{i}, \quad\left[S_{\alpha}{ }^{i}\right]=L^{1 / 2}$

Bringing these decompositions into the algebra (B.5) we obtain

$$
\begin{aligned}
& {\left[M_{a b}, M_{c d}\right]=\eta_{a[c} M_{d] b}-\eta_{b[c} M_{d] a},} \\
& {\left[P_{a}, M_{c d}\right]=\eta_{a[b} P_{c]},} \\
& {\left[D, P_{a}\right]=P_{a},} \\
& {\left[K_{a}, M_{c d}\right]=\eta_{a[b} K_{c]},} \\
& {\left[P_{a}, K_{b}\right]=2\left(\eta_{a b} D+2 M_{a b}\right),} \\
& {\left[M_{a b}, Q_{\alpha}^{i}\right]=-\frac{1}{4}\left(\hat{\gamma}_{a b} Q^{i}\right)_{\alpha},} \\
& {\left[K_{a}, Q_{\alpha}{ }^{i}\right]=-\left(\hat{\gamma}_{a T} S^{i}\right)_{\alpha},} \\
& {\left[D, Q_{\alpha}{ }^{i}\right]=\frac{1}{2} Q_{\alpha}{ }^{i},} \\
& \left\{Q_{\alpha}{ }^{i}, \bar{Q}_{j}{ }^{\beta}\right\}=\delta_{j}{ }^{i}\left(\hat{\gamma}^{a T}\right)_{\alpha}{ }^{\beta} P_{a}, \\
& {\left[D, K_{a}\right]=-K_{a},} \\
& \left\{Q_{\alpha}{ }^{i}, \bar{S}_{j}{ }^{\beta}\right\}=\delta_{j}{ }^{i}\left(\hat{\gamma}^{a b}\right)_{\alpha}{ }^{\beta} M_{a b}+\delta_{j}{ }^{i} \delta_{\alpha}{ }^{\beta} D-2 \delta_{\alpha}{ }^{\beta} U_{j}{ }^{i} .
\end{aligned}
$$

By having given appropriate dimensions to the generators, this algebra contains no dimensionful constants as opposed to the AdS-decomposition where it was unavoidable.

A superconformal object can be decomposed as follows

$$
A=A_{P}^{a} P_{a}+A_{M}^{a b} M_{a b}+A_{D} D+A_{K}^{a} K_{a}+A_{i}{ }^{j} U_{j}{ }^{i}+\left(\bar{A}_{Q i}{ }^{\alpha} Q_{\alpha}{ }^{i}+\bar{A}_{S i}{ }^{\alpha} S_{\alpha}{ }^{i}+\text { h.c. }\right) \text {, }
$$

and this yields the following relations

$$
\begin{aligned}
A_{P}^{a} & =\frac{R}{2}\left(\hat{A}^{a T}+\hat{A}^{a S}\right)=\frac{1}{2}\left(\tilde{A}^{a}+R \tilde{A}^{a S}\right), \\
A_{M}^{a b} & =\hat{A}^{a b}=\tilde{A}^{a b}
\end{aligned}
$$




$$
\begin{aligned}
A_{D} & =\hat{A}^{T S}=-R^{-1} \tilde{A}^{S}, \\
A_{K}^{a} & =\frac{1}{2 R}\left(\hat{A}^{a T}-\hat{A}^{a S}\right)=\frac{1}{2 R^{2}}\left(\tilde{A}^{a}-R \tilde{A}^{a S}\right) \\
A_{i}{ }^{j} & =\hat{A}_{i}{ }^{j}=\tilde{A}_{i}{ }^{j} \\
\bar{A}_{Q i}{ }^{\alpha} & =\frac{R^{1 / 2}}{\sqrt{2}} \overline{\hat{A}}_{i}{ }^{\hat{\beta}}\left(\mathcal{P}_{Q}\right)_{\hat{\beta}}{ }^{\hat{\alpha}}=\frac{1}{\sqrt{2}} \overline{\tilde{A}}_{i}{ }^{\hat{\beta}}\left(\mathcal{P}_{Q}\right)_{\hat{\beta}}{ }^{\hat{\alpha}} \\
\bar{A}_{S i}{ }^{\alpha} & =\frac{R^{-1 / 2}}{\sqrt{2}} \overline{\hat{A}}_{i}{ }^{\hat{\beta}}\left(\mathcal{P}_{S}\right)_{\hat{\beta}}{ }^{\hat{\alpha}}=\frac{1}{\sqrt{2} R} \overline{\tilde{A}}_{i}{ }^{\hat{\beta}}\left(\mathcal{P}_{S}\right)_{\hat{\beta}}{ }^{\hat{\alpha}} .
\end{aligned}
$$

It is interesting to note that the translations and the special conformal transformations in the conformal decomposition mix the AdS translations and structure group rotations.

To conclude this section we give the AdS objects in terms of the their conformal counterparts.

$$
\begin{aligned}
& \tilde{A}^{a}=A_{P}^{a}+R^{2} A_{K}^{a}, \\
& \tilde{A}^{S}=-R A_{D}, \\
& \tilde{A}^{a S}=R^{-1} A_{P}^{a}-R A_{K}^{a}, \\
& \tilde{A}^{a b}=A_{M}^{a b} \\
& \tilde{A}_{\hat{\alpha}}^{i}=\sqrt{2}\left(\begin{array}{c}
A_{Q \alpha}{ }^{i} \\
R A_{S \alpha}{ }^{i}
\end{array}\right),
\end{aligned}
$$

where just for notational reasons we have a basis in which $\hat{\gamma}_{S T}$ is diagonal.

\section{C $\quad A d S_{5} \times S^{5}$ as a coset space}

Our aim in this section is to construct the coset space $A d S_{5} \times S^{5}$. First we consider $A d S_{5}$ as a coset space and then we discuss the $S^{5}$ coset space. We conclude this section with a discussion of an appropriate choice of fermionic coordinates for the coset superspace.

\section{C.1 $A d S_{5}$ as a coset space}

The $A d S_{5}$ space is the coset

$$
A d S_{5}=\frac{\mathrm{SO}(2,4)}{\mathrm{SO}(1,4)}
$$

The algebra to be considered is the bosonic part of the algebra in appendix B.1 (ignoring the internal part). We choose horospherical coordinates,

$$
d s^{2}=\rho^{2} d x^{2}+\left(\frac{R}{\rho}\right)^{2} d \rho^{2}
$$


where the boundary is parametrized by $x^{m}$ and is at $\rho=\infty$. The coset representative for horospherical coordinates is given in the spinor representation of $\mathrm{SO}(2,4)$. It can be derived from the supergravity Killing spinor [11] and can be written as

$$
v\left(\tilde{x}^{\tilde{m}}\right)=v_{\operatorname{conf}}(x)\left(\rho^{-1 / 2} \frac{1}{2}\left(1-\hat{\gamma}_{S T}\right)+\rho^{1 / 2} \frac{1}{2}\left(1+\hat{\gamma}_{S T}\right)\right),
$$

where $v_{\text {conf }}(x)$ is the coset representative of the 4-dimensional conformal Minkowski space

$$
v_{\text {conf }}(x)=1+\frac{x^{m}}{R} \hat{\gamma}_{m T} \frac{1}{2}\left(1+\hat{\gamma}_{S T}\right) .
$$

The flat $S$ direction is related to the bulk direction $\rho$ of $A d S_{5}$. Straightforward computation gives the Cartan forms (2.1)

$$
v^{-1} d v \equiv L^{\Lambda} T_{\Lambda}=e^{\tilde{m}} P_{\tilde{m}}+\omega^{\tilde{m} \tilde{n}} M_{\tilde{m} \tilde{n}}
$$

with non-vanishing components

$$
e^{m}=d x^{m} \rho, \quad e^{\rho}=d \rho \frac{R}{\rho}, \quad \omega^{m \rho}=d x^{m} \frac{\rho}{R} .
$$

The Killing fields $\Sigma_{0}(2.10)$ are determined by an $\tilde{x}$-independent $\mathrm{SO}(2,4)$ object,

$$
\Upsilon=\tilde{a}^{\tilde{m}} \tilde{P}_{\tilde{m}}+\tilde{\lambda}_{M}^{\tilde{m} \tilde{n}} \tilde{M}_{\tilde{m} \tilde{n}}
$$

Using the AdS-decomposition

$$
\tilde{P}_{\tilde{m}}=\frac{2}{R} \hat{M}_{\tilde{m} T}, \quad \tilde{M}_{\tilde{m} \tilde{n}}=\hat{M}_{\tilde{m} \tilde{n}}
$$

yields the $A d S_{5}$ Killing fields

$$
\begin{aligned}
\Sigma_{0}^{m} & =\rho \xi^{m}(x)+\frac{R^{2}}{\rho} \lambda_{(K)}^{m}, & \Sigma_{0}^{\rho} & =-\Lambda_{D}(x) R, \\
\Sigma_{0}^{m \rho} & =\frac{\rho}{R} \xi^{m}(x)-\frac{R}{\rho} \Lambda_{(K)}^{m}, & \Sigma_{0}^{m n} & =\Lambda_{M}^{m n}(x),
\end{aligned}
$$

where the Killing fields have been written in terms of the conformal parameters. From these we get the Killing vectors and compensating (stability group) transformations. The Killing vectors (2.12) are given by

$$
\begin{aligned}
\xi^{m} & =\hat{\Lambda}_{-}^{m}+\hat{\Lambda}^{m n} x_{n}+\hat{\Lambda}_{+}^{+} x^{m}+\left(x^{2} \hat{\Lambda}_{+}^{m}-2 x^{m} x_{n} \hat{\Lambda}_{+}^{n}\right)+\frac{R^{2}}{\rho^{2}} \hat{\Lambda}_{+}^{m}, \\
\xi^{\rho} & =-\hat{\Lambda}_{+}^{+}-2 x_{m} \hat{\Lambda}_{+}^{m} .
\end{aligned}
$$

in terms of the independent parameters $\hat{\Lambda}_{-}^{m}, \hat{\Lambda}^{m n}, \hat{\Lambda}_{+}^{+}$and $\hat{\Lambda}_{+}^{m}$, and the compensating transformations are

$$
l^{m n}=\Lambda_{(M)}^{m n}, \quad l^{m \rho}=-2 \frac{R}{\rho} \lambda_{(K)}^{m} .
$$


We can recast the Killing vectors into a form related to the conformal isometries on $x^{m}$. We define

$$
a^{m}=\hat{\Lambda}_{-}^{m}, \quad \lambda_{(M)}^{m n}=\hat{\Lambda}^{m n}, \quad \lambda_{D}=\hat{\Lambda}_{+}^{+}, \quad \lambda_{(K)}^{m}=\hat{\Lambda}_{+}^{m},
$$

and obtain

$$
\delta x^{m}=-\xi_{C}^{m}(x)-\frac{R^{2}}{\rho^{2}} \lambda_{(K)}^{m}, \quad \delta \rho=\Lambda_{D}(x) \rho
$$

with

$$
\begin{aligned}
\xi_{C}^{m}(x) & =a^{m}+\lambda_{(M)}^{m n}+\lambda_{D} x^{m}+\left(x^{2} \lambda_{(K)}^{m}-2 x^{m} x \cdot \lambda_{(K)}\right), \\
\Lambda_{(M)}^{m n}(x) & =\lambda_{(M)}^{m n}-4 x^{[m} \lambda_{(K)}^{n]}, \\
\Lambda_{D}(x) & =\lambda_{D}-2 \lambda_{(K)} \cdot x .
\end{aligned}
$$

Where $a^{m}, \lambda_{(M)}^{m n}, \lambda_{D}$ and $\lambda_{(K)}^{m}$ are the constant parameters of translations, Lorentz rotations, dilatations and special conformal transformations for the conformal space in four dimensions, spanned by the coordinates $x^{m}$. We have include the $C$ as a subscript for $\xi_{C}^{m}$ to stress that it is expressed in terms of the conformal parameters.

\section{C.2 $\quad S^{5}$ as a coset space}

The sphere is the coset space

$$
S^{5}=\frac{\mathrm{SO}(6)}{\mathrm{SO}(5)}
$$

The algebra to be considered is the $\mathrm{SO}(6)$ algebra

$$
\left[\hat{M}_{\hat{m}^{\prime} \hat{n}^{\prime}}^{\prime}, \hat{M}_{\hat{p}^{\prime} \hat{q}^{\prime}}^{\prime}\right]=\delta_{\hat{m}^{\prime}\left[\hat{p}^{\prime}\right.} \hat{M}_{\left.\hat{q}^{\prime}\right] \hat{n}^{\prime}}^{\prime}-\delta_{\hat{n}^{\prime}\left[\hat{p}^{\prime}\right.} \hat{M}_{\left.\hat{q}^{\prime}\right] \hat{m}^{\prime}}^{\prime}
$$

where in the sphere decomposition

$$
\tilde{P}_{m^{\prime}}^{\prime}=\frac{2}{R} \hat{M}_{m^{\prime} S^{\prime}}^{\prime}, \quad \tilde{M}_{m^{\prime} n^{\prime}}^{\prime}=\hat{M}_{m^{\prime} n^{\prime}}^{\prime}
$$

with $m^{\prime}$ the 5 flat tangent directions of the sphere.

We will work in stereographic coordinates $z^{m^{\prime}}$

$$
d s^{2}=\frac{4 R^{2}}{\left(1+z^{2}\right)^{2}} d z^{2},
$$

where $z^{2}=z^{m^{\prime}} \eta_{m^{\prime} n^{\prime}} z^{n^{\prime}}$. The convenient coset representative for the sphere in these coordinates is

$$
u\left(z^{m^{\prime}}\right)=\left(1+z^{2}\right)^{-1 / 2}\left(1+z^{m^{\prime}} \hat{\gamma}_{m^{\prime} S^{\prime}}^{\prime}\right)
$$

given in the spinor representation

$$
\hat{M}_{\hat{m}^{\prime} \hat{n}^{\prime}}^{\prime}=\frac{1}{4} \hat{\gamma}_{\hat{m}^{\prime} \hat{n}^{\prime}}^{\prime}
$$


where the matrices $\hat{\gamma}_{\hat{m}^{\prime} \hat{n}^{\prime}}^{\prime}$ are elements of the $\mathrm{SO}(6)$ Clifford algebra. Straightforward computation gives the Cartan forms (2.1)

$$
u^{-1} d u=e^{m^{\prime}} P_{m^{\prime}}+\omega^{m^{\prime} n^{\prime}} M_{m^{\prime} n^{\prime}}
$$

with

$$
e^{m^{\prime}}=2 R \frac{d z^{m^{\prime}}}{1+z^{2}}, \quad \omega^{m^{\prime} n^{\prime}}=4 \frac{z^{\left[m^{\prime}\right.} d z^{\left.n^{\prime}\right]}}{1+z^{2}}
$$

We introduce the rigid $\mathrm{SO}(6)$-valued parameter $\Upsilon_{S}=\Lambda^{\hat{m}^{\prime} \hat{n}^{\prime}} \hat{M}_{\hat{m}^{\prime} \hat{n}^{\prime}}^{\prime}$ and derive the Killing field (2.12),

$$
\begin{aligned}
\Sigma_{0}^{m^{\prime}} & =\frac{2 R}{1+z^{2}}\left(\frac{1}{2}\left(1-z^{2}\right) \Lambda^{m^{\prime} S^{\prime}}+\Lambda^{m^{\prime} n^{\prime}} z_{n^{\prime}}+z^{m^{\prime}} z_{n^{\prime}} \Lambda^{n^{\prime} S^{\prime}}\right), \\
\Sigma_{0}^{m^{\prime} n^{\prime}} & =\Lambda^{m^{\prime} n^{\prime}}+\frac{4}{1+z^{2}}\left(z^{\left[m^{\prime}\right.} \Lambda^{\left.n^{\prime}\right] S^{\prime}}+z^{\left[m^{\prime}\right.} \Lambda^{\left.n^{\prime}\right] p^{\prime}} z_{p^{\prime}}\right),
\end{aligned}
$$

leading to the isometries

$$
-\delta z^{m^{\prime}}=\xi^{m^{\prime}}=\frac{1}{2}\left(1-z^{2}\right) \Lambda^{\prime m^{\prime} S^{\prime}}+\Lambda^{\prime m^{\prime} n^{\prime}} z_{n^{\prime}}+z^{m^{\prime}} z_{n^{\prime}} \Lambda^{\prime n^{\prime} S} .
$$

\section{C.3 $\quad A_{d} S_{5} \times S^{5}$ and adapted fermionic coordinates}

The bosonic space is of a direct product form

$$
A d S_{5} \times S^{5}
$$

The bosonic coset representative $g(X)$ then also takes the form of a direct product $g(X)=$ $v \otimes u$, in terms of the bosonic representatives for AdS and $S$ obtained before. We can enlarge this bosonic space to a superspace by the coset construction. We already derived the representatives for the bosonic subspaces. The only thing that is lacking is the fermionic coordinate choice, encoded in the matrix $e_{\dot{\alpha}}{ }^{\alpha}$.

The conformal structure of the AdS boundary and associated isometries is most apparent in the horospherical coordinates. The coordinates $x^{m}$, which parametrize the directions parallel to the boundary $\rho \rightarrow \infty$, can then be identified with the coordinates $x^{m}$ of the conformal Minkowski space. To continue this, we would like that half of the anticommuting coordinates of the $A d S \times S$ superspace can be identified with the $\theta$ 's of the conformal superspace. This can be done by appropriately considering the relation between the AdS and conformal decompositions. As a coordinate choice we will take

$$
\begin{aligned}
\Theta & =\bar{\Theta}_{i} \mathcal{Q}^{i}+\overline{\mathcal{Q}}_{i} \Theta^{i} \\
& =\left(u^{-1}\right)_{i}{ }^{j} \bar{\theta}_{j} \rho^{1 / 2} Q^{i}+\left(u^{-1}\right)_{i}{ }^{j} \bar{\vartheta}_{j} \rho^{-1 / 2} S^{i}+\bar{Q}_{i} \rho^{1 / 2} \theta^{j} u_{j}{ }^{i}+\bar{S}_{i} \rho^{-1 / 2} \vartheta^{j} u_{j}{ }^{i} .
\end{aligned}
$$

The two coordinates $\{\theta, \vartheta\}$ together build up the anticommuting coordinate of the $A d S \times S$ superspace $\tilde{\theta}$ by

$$
\tilde{\theta}_{\hat{\alpha}}^{i}=\sqrt{2}\left(\begin{array}{c}
\theta_{\alpha}^{i} \\
R \vartheta_{\alpha}^{i}
\end{array}\right) .
$$


We will call these coordinates the super-horospherical coordinates

$$
Z^{M}=\left\{x^{m}, \rho, z^{m^{\prime}}, \theta^{i}, \vartheta^{i}\right\} .
$$

The parametrization for the fermionic symmetry parameter $\varepsilon$ will be

$$
\begin{aligned}
\bar{\varepsilon}_{i}^{\alpha} & =\frac{1}{\sqrt{2}}\left(u^{-1}\right)_{i}{ }^{j}\left[R \rho^{-1 / 2} \bar{\eta}_{j}^{\hat{\beta}} \frac{1}{2}\left(1-\hat{\gamma}_{S T}\right)_{\hat{\beta}}{ }^{\alpha}+\rho^{1 / 2}\left(\bar{\epsilon}_{j}{ }^{\hat{\beta}}+\bar{\eta}_{j}{ }^{\hat{\gamma}}\left(\hat{\gamma}_{m T}\right)_{\hat{\gamma}}{ }^{\hat{\beta}} x^{m}\right) \frac{1}{2}\left(1+\hat{\gamma}_{S T}\right)_{\hat{\beta}}{ }^{\alpha}\right] \\
& =\frac{1}{\sqrt{2}}\left(u^{-1}\right)_{i}{ }^{j}\left[R \rho^{-1 / 2} \bar{\eta}_{j}^{\beta} \frac{1}{2}\left(1+\gamma_{5}\right)_{\beta}{ }^{\alpha}+\rho^{1 / 2}\left(\bar{\epsilon}_{j}{ }^{\beta}-\bar{\eta}_{j}{ }^{\gamma}\left(\gamma_{m}\right)_{\gamma}{ }^{\beta} x^{m}\right) \frac{1}{2}\left(1-\gamma_{5}\right)_{\beta}{ }^{\alpha}\right] .
\end{aligned}
$$

This is determined by Killing spinor equation and its solution [11, 19]. We can make the same super-horospherical decomposition for the $\kappa$-symmetry parameter

$$
\kappa_{+}^{\alpha} \mathbf{K}_{\alpha}=\left(\rho^{1 / 2} u_{i}^{j} \bar{Q}_{j} \kappa_{+Q}^{i}+\text { h.c. }\right)+\left(\rho^{-1 / 2} u_{i}^{j} \bar{S}_{j} \kappa_{+S}^{i}+\text { h.c. }\right),
$$

and the relationship between its irreducible components is modified with factors of $R$ such that $\kappa_{+Q}=R \beta_{-} \kappa_{+S}$, or equivalently, $\kappa_{+S}=-\frac{1}{R} \beta_{+} \kappa_{+Q}$.

Open Access. This article is distributed under the terms of the Creative Commons Attribution License (CC-BY 4.0), which permits any use, distribution and reproduction in any medium, provided the original author(s) and source are credited.

\section{References}

[1] E. Bergshoeff, F. Coomans, R. Kallosh, C.S. Shahbazi and A. Van Proeyen, Dirac-Born-Infeld-Volkov-Akulov and deformation of supersymmetry, JHEP 08 (2013) 100 [arXiv: 1303.5662] [INSPIRE].

[2] A. Van Proeyen, Superconformal tensor calculus in $N=1$ and $N=2$ supergravity, CERN-TH-3579 (1983) [INSPIRE].

[3] M. Kaku, P.K. Townsend and P. van Nieuwenhuizen, Gauge theory of the conformal and superconformal group, Phys. Lett. B 69 (1977) 304 [INSPIRE].

[4] S. Ferrara, M. Kaku, P.K. Townsend and P. van Nieuwenhuizen, Gauging the graded conformal group with unitary internal symmetries, Nucl. Phys. B 129 (1977) 125 [INSPIRE].

[5] M. Kaku, P.K. Townsend and P. van Nieuwenhuizen, Properties of conformal supergravity, Phys. Rev. D 17 (1978) 3179 [INSPIRE].

[6] M. Kaku and P.K. Townsend, Poincaré supergravity as broken superconformal gravity, Phys. Lett. B 76 (1978) 54 [INSPIRE].

[7] D.Z. Freedman and A. Van Proeyen, Supergravity, Cambridge University Press, Cambridge U.K. (2012).

[8] P. Claus, R. Kallosh and A. Van Proeyen, $M$ five-brane and superconformal $(0,2)$ tensor multiplet in six-dimensions, Nucl. Phys. B 518 (1998) 117 [hep-th/9711161] [INSPIRE].

[9] P. Claus, R. Kallosh, J. Kumar, P.K. Townsend and A. Van Proeyen, Conformal theory of M2, D3, M5 and D1-branes + D5-branes, JHEP 06 (1998) 004 [hep-th/9801206] [INSPIRE]. 
[10] P. Claus et al., Black holes and superconformal mechanics, Phys. Rev. Lett. 81 (1998) 4553 [hep-th/9804177] [INSPIRE].

[11] P. Claus and R. Kallosh, Superisometries of the AdS $\times S$ superspace, JHEP 03 (1999) 014 [hep-th/9812087] [INSPIRE].

[12] P. Claus, Near horizon supergravity superspace, Lect. Notes Phys. 525 (1999) 375 [hep-th/9812220] [INSPIRE].

[13] R. Kallosh, J. Rahmfeld and A. Rajaraman, Near horizon superspace, JHEP 09 (1998) 002 [hep-th/9805217] [INSPIRE].

[14] L. Castellani, R. D'Auria and P. Fre, Supergravity and superstrings: a geometric perspective. Volume 1: mathematical foundations, World Scientific, Singapore (1991).

[15] G.T. Horowitz and A. Strominger, Black strings and P-branes, Nucl. Phys. B 360 (1991) 197 [inSPIRE].

[16] E. Bergshoeff and P.K. Townsend, Super D-branes, Nucl. Phys. B 490 (1997) 145 [hep-th/9611173] [INSPIRE].

[17] E. Bergshoeff and P.K. Townsend, Super D-branes revisited, Nucl. Phys. B 531 (1998) 226 [hep-th/9804011] [INSPIRE].

[18] R.R. Metsaev and A.A. Tseytlin, Supersymmetric D3-brane action in $A d S_{5} \times S^{5}$, Phys. Lett. B 436 (1998) 281 [hep-th/9806095] [INSPIRE].

[19] H. Lü, C.N. Pope and J. Rahmfeld, A construction of Killing spinors on $S^{n}$, J. Math. Phys. 40 (1999) 4518 [hep-th/9805151] [INSPIRE]. 